\title{
Identification of Parameters through the Approximate Periodic Solutions of a Parabolic System
}

\author{
Ling Lei \\ Department of Mathematics, Zhejiang University, \\ Hangzhou, 310027, P.R.China
}

\begin{abstract}
This work is concerned with the identification problem for what we call the perturbation term or error term in a parabolic partial differential equation, through its approximate periodic solutions. The observation is made over a subregion of the physical domain. The existence and uniqueness problem of the approximate periodic solutions is studied in the first part of the paper. A solution to the identification problem is given in the second part of the paper. The main ingredients to be used include the classical Garlerkin method and the more recently developed Carleman estimates for a parabolic system.
\end{abstract}

Key words. Identification of parameter, Garlerkin method, Carleman inequality, approximate periodic solution, parabolic equation.

AMS subject classification. 35K99, 93A99.

\section{Introduction}

Let $\Omega \subset \mathbf{R}^{n}$ be a bounded domain with $C^{2}$-smooth boundary $\partial \Omega$ and let $\omega \subset \Omega$ be a subdomain. Write $Q=\Omega \times(0, T)$ with $T>0$ and write $Q^{\omega}=\omega \times(0, T)$. Consider the following parabolic equation:

$$
\begin{cases}\frac{\partial u}{\partial t}(x, t)+L u(x, t)=f(x, t), & \text { in } \quad Q=\Omega \times(0, T), \\ u(x, t)=0, & \text { on } \Sigma=\partial \Omega \times(0, T),\end{cases}
$$

where

$$
\begin{gathered}
L u(x, t)=L_{0} u(x, t)+e(x, t) u(x, t), \\
L_{0} u(x, t)=-\sum_{i, j=1}^{n} D_{j}\left(a^{i j}(x) D_{i} u(x, t)\right)+\sum_{i=1}^{n} b^{i}(x) D_{i} u(x, t)-\sum_{i=1}^{n} D_{i}\left(b^{i}(x) u(x, t)\right)+c(x) u(x, t) .
\end{gathered}
$$


Here and in what follows, we write $D_{j}=\frac{\partial}{\partial x_{j}}$. We also use the standard summation convection. Namely, repeated indices imply summation from 1 to $n$. Throughout of the paper, we make the following regularity assumptions for the coefficients:

$(\mathrm{I}): a^{i j}(x) \in \operatorname{Lip}(\bar{\Omega}), a^{i j}(x)=a^{j i}(x)$, and $\lambda^{*}|\xi|^{2} \leq a^{i j}(x) \xi_{i} \xi_{j} \leq \frac{1}{\lambda^{*}}|\xi|^{2}$, for $\xi \in \mathbf{R}^{n}$ with $\lambda^{*}$ a certain positive constant;

$(\mathrm{II}): b^{i}(x) \in \operatorname{Lip}(\bar{\Omega}), c(x) \in L^{\infty}(\Omega)$, and $e(x, t) \in L^{\infty}\left(0, T ; L^{q}(\Omega)\right)$ with $q>\frac{n+2}{2}$ and $f(x, t) \in L^{2}(Q)$.

In many applications, one often encounters various problems, such as the inverse problem and the Pontryagin maximum principle, related to the periodic solutions of (1.1). (See [11] [15] [16] [17], etc). Here, we recall that a periodic solution of (1.1) is a solution satisfying the following condition:

$$
u(x, 0)=u(x, T) \text { in } \Omega .
$$

In (1.1), the coefficients of the principal part $L_{0}$ of the operator $L$ is $t$-independent. However, the one for what we call the perturbation term or the error term $e(x, t)$ may well depend on the time variable $t$. It is known that for (1.1), when the operator $L$ is not positive (for instance, when $e(x, t)$ takes negative values), the periodic solution of (1.1) may not exist for a generic choice of $f(x, t)$. (See Example 3.4 in Section 3). Namely, adding an error term with coefficient $e(x, t)$ to the system may well destroy the periodicity of certain solutions even if $L_{0}$ is a positive operator. However, as we will show, the system always possesses solutions with certain approximate periodicity. This makes it a natural problem to consider the inverse problem, the Pontryagin problem, and many others, for (1.1) through a certain family of solutions with approximate periodicity. In this paper, we will make an effort towards this study by introducing the concept of approximate periodic solutions through the principle part $L_{0}$ of $L$. We then study the existence and uniqueness of such solutions and use them to identify the error term through the observation of solutions over $\omega$.

We next introduce the concept of $\mathcal{K}$-approximate periodic solutions of $(1.1)$, where $\mathcal{K}$ is a non-negative integer.

First, we notice that $L_{0}$ is a symmetric operator. Consider the eigenvalue problem of $L_{0}$ :

$$
\left\{\begin{array}{l}
L_{0} v(x)=\lambda v(x), \\
\left.v(x)\right|_{\partial \Omega}=0 .
\end{array}\right.
$$

It is well-known (see [8]) that (1.3) has a complete set of eigenvalues $\left\{\lambda_{j}\right\}_{j=1}^{\infty}$ with the associate eigenvectors $\left\{X_{j}(x)\right\}_{j=1}^{\infty}$ such that $L_{0} X_{j}(x)=\lambda_{j} X_{j}(x),-\infty<\lambda_{1} \leq \lambda_{2} \leq$ $\cdots \leq \lambda_{j} \leq \cdots<\infty, \lim _{j \rightarrow \infty} \lambda_{j}=\infty, X_{j}(x) \in H_{0}^{1}(\Omega)$. Choose $\left\{X_{j}(x)\right\}_{j=1}^{\infty}$ so that it serves an orthonormal basis of $L^{2}(\Omega)$. Therefore, $\forall u(x, t) \in L^{2}(Q)$, we have $u(x, t)=$ $\sum_{j=1}^{\infty} u_{j}(t) X_{j}(x)$, where $u_{j}(t)=\int_{\Omega} u(x, t) X_{j}(x) d x \in L^{2}(0, T)$. 
Definition 1.1. We call $u(x, t)$ is a $\mathcal{K}$-approximate periodic solution of (1.1) with respect to its principal part $L_{0}$ if

(a): $u \in C\left([0, T] ; L^{2}(\Omega)\right) \cap L^{2}\left(0, T ; H_{0}^{1}(\Omega)\right)$ is a weak solution of (1.1);

(b): $u \in \mathcal{S}_{\mathcal{K}}=\left\{u \in C\left([0, T] ; L^{2}(\Omega)\right) ; u_{j}(0)=u_{j}(T)\right.$ for $\left.j \geq \mathcal{K}+1, u_{j}(t)=\int_{\Omega} u(x, t) X_{j}(x) d x\right\}$.

Here, we recall that $u(x, t)$ is said to be a weak solution of (1.1) with the initial value $u(x, 0)=\psi(x)$ if $u \in C\left([0, T] ; L^{2}(\Omega)\right) \cap L^{2}\left(0, T ; H_{0}^{1}(\Omega)\right)$ and for any testing function $\varphi \in$ $H_{0}^{1,1}(Q)=\left\{h \in L^{2}(Q) ; \partial_{t} h \in L^{2}(Q), D_{i} h \in L^{2}(Q)\right.$ for all $\left.i=1,2 \cdots n,\left.h(x, t)\right|_{\partial \Omega}=0\right\}$, we have, for all $t$,

$$
(u(\cdot, t), \varphi(\cdot, t))-\int_{0}^{t}\left(u, \varphi_{\tau}\right) d \tau+\int_{0}^{t}(L u, \varphi) d \tau=(\psi, \varphi(\cdot, 0))+\int_{0}^{t}(f, \varphi) d \tau .
$$

When $\mathcal{K}=0$, we will always regard $\sum_{j=1}^{0}=0$. Hence, a 0 -approximate periodic solution of (1.1) is a regular periodic solution.

It should be mentioned that in the above definition, we need only to assume that $u(x, t) \in L^{\infty}\left(0, T ; L^{2}(\Omega)\right) \cap L^{2}\left(0, T ; H_{0}^{1}(\Omega)\right)$ to start with. Then it holds automatically that $u \in C\left([0, T] ; L^{2}(\Omega)\right)$ (see Chapter 3 of [5]). Also, we notice that

$u \in \mathcal{S}_{\mathcal{K}} \Longleftrightarrow\left(u-\sum_{j=1}^{\mathcal{K}}\left(u, X_{j}\right) X_{j}\right)(x, 0)=\left(u-\sum_{j=1}^{\mathcal{K}}\left(u, X_{j}\right) X_{j}\right)(x, T)$.

In the above formula and in what follows, we write $(u(\cdot, t), \varphi(\cdot, t))=\int_{\Omega} u(x, t) \varphi(x, t) d x$, $(u(\cdot, t), u(\cdot, t))=\|u(\cdot, t)\|^{2}$, and we denote $u_{t}$ for the derivative of $u(x, t)$ with respect to $t$.

Our first result of this paper can be stated as follows:

Theorem 1.2. Let $e(x, t) \in \mathcal{M}_{q}=\left\{e(x, t) \in L^{\infty}\left(0, T ; L^{q}(\Omega)\right) ;\right.$ ess $\sup _{t \in(0, T)}\|e(x, t)\|_{L^{q}(\Omega)}$ $\leq M, q>\frac{n+2}{2}, M$ is a constant $\}$. Then, there exists an integer $\mathcal{K}_{0} \equiv \mathcal{K}_{0}\left(L_{0}, M, \Omega\right) \geq 0$ such that for any $\mathcal{K} \geq \mathcal{K}_{0}$ and any initial value $a_{I}=\left(a_{1}, a_{2}, \cdots, a_{\mathcal{K}}\right) \in \mathbf{R}^{\mathcal{K}}$, we have a unique solution to the following equation:

$$
\begin{cases}\frac{\partial u(x, t)}{\partial t}+L_{0} u(x, t)+e(x, t) u(x, t)=f(x, t), & \text { in } Q, \\ u(x, t)=0, & \text { on } \Sigma, \\ \left(u(x, 0), X_{j}(x)\right)=a_{j}, & \text { for } j \leq \mathcal{K}, \\ u \in \mathcal{S}_{\mathcal{K}} . & \end{cases}
$$

Moreover, for such a solution $u(x, t)$, we have the following energy estimate:

$$
\sup _{t \in[0, T]}\|u(\cdot, t)\|^{2}+\int_{0}^{T}\|\nabla u(\cdot, t)\|^{2} d t \leq C\left(L_{0}, M, \Omega\right)\left(\left|a_{I}\right|^{2}+\int_{Q} f^{2} d x d t\right) .
$$

The second part of this work is to study an inverse problem. We will identify $\left(e(x, t), a_{I}\right)$ from $\mathcal{M}_{q} \times \mathbf{R}^{\mathcal{K}}$ via the observation of solutions for (1.4) on the subdomain $\omega \subset \Omega$. More precisely, we shall study the following identification problem: 
Problem $(P)$ Find the minimum value of $\int_{Q^{\omega}}|u-\widetilde{u}|^{2} d x d t$ for $\left(e(x, t), a_{I}\right) \in \mathcal{M}_{q} \times \mathbf{R}^{\mathcal{K}}$ with $u$ satisfying

$$
\begin{cases}\frac{\partial u(x, t)}{\partial t}+L_{0} u(x, t)+e(x, t) u(x, t)=f(x, t), & \text { in } Q, \\ u(x, t)=0, & \text { on } \Sigma, \\ \left(u(x, 0), X_{j}(x)\right)=a_{j}, & \text { for } j \leq \mathcal{K} \\ u \in \mathcal{S}_{\mathcal{K}}, & \end{cases}
$$

where $\widetilde{u} \in L^{2}\left(Q^{\omega}\right)$ is a given function.

Making use of Theorem 1.2 and the Carleman inequality established in [2] [11] [16], etc, we are able to prove the existence of solutions to problem $(P)$. Our second main result can be stated as follows:

Theorem 1.3. Let $\mathcal{K}$ be as in Theorem 1.2. Then there exist an $e^{*}(x, t) \in \mathcal{M}_{q}$ and $a_{I}^{*} \in \mathbf{R}^{\mathcal{K}}$ such that

$$
\int_{Q^{\omega}}\left|u\left(e^{*}, a_{I}^{*} ; x, t\right)-\widetilde{u}\right|^{2} d x d t=\inf _{\left(e, a_{I}\right) \in \mathcal{M}_{q} \times \mathbf{R}^{\mathcal{K}}} \int_{Q^{\omega}}\left|u\left(e, a_{I} ; x, t\right)-\widetilde{u}\right|^{2} d x d t .
$$

Here $\widetilde{u} \in L^{2}\left(Q^{\omega}\right)$ is a given function and $u\left(e, a_{I} ; x, t\right)$ is the solution of equation (1.4) with error coefficient $e(x, t)$ and $\left(u\left(e, a_{I} ; \cdot, 0\right), X_{j}(\cdot)\right)=a_{j}$ for $j \leq \mathcal{K}$, where $a_{I}=\left(a_{1}, \cdots, a_{\mathcal{K}}\right) \in$ $\mathbf{R}^{\mathcal{K}}$.

Theorem 1.3 can be immediately used to give the following slightly more general result:

Corollary 1.4. Let $k$ be a non-negative integer. Then there exist an $e^{*}(x, t) \in \mathcal{M}_{q}$ and $a_{I}^{*} \in \mathbf{R}^{k}$ such that

$$
\int_{Q^{\omega}}\left|u^{*}-\widetilde{u}\right|^{2} d x d t=\inf _{\left(e, a_{I}\right) \in \mathcal{M}_{q} \times \mathbf{R}^{k}, u \in U\left(e, a_{I} ; x, t\right)} \int_{Q^{\omega}}|u-\widetilde{u}|^{2} d x d t
$$

Here $\widetilde{u} \in L^{2}\left(Q^{\omega}\right)$ is a given function and $U\left(e, a_{I} ; x, t\right)$ is the set of solutions of the following equation, which we assume to be non-empty:

$$
\begin{cases}\frac{\partial u(x, t)}{\partial t}+L_{0} u(x, t)+e(x, t) u(x, t)=f(x, t), & \text { in } Q, \\ u(x, t)=0, & \text { on } \Sigma, \\ \left(u(x, 0), X_{j}(x)\right)=a_{j}, a_{I}=\left(a_{1}, \cdots, a_{k}\right), & \text { for } j \leq k \\ u \in \mathcal{S}_{k} . & \end{cases}
$$


Similarly, $u^{*}$ satisfies the following equation:

$$
\begin{cases}\frac{\partial u^{*}(x, t)}{\partial t}+L_{0} u^{*}(x, t)+e^{*}(x, t) u^{*}(x, t)=f(x, t), & \text { in } Q, \\ u^{*}(x, t)=0, & \text { on } \Sigma, \\ \left(u^{*}(x, 0), X_{j}(x)\right)=a_{j}^{*}, a_{I}^{*}=\left(a_{1}^{*}, \cdots, a_{k}^{*}\right), & \text { for } j \leq k, \\ u^{*} \in \mathcal{S}_{k} . & \end{cases}
$$

Notice that in Corollary 1.4, (1.6) may have a family of different solutions.

It is not clear to us if the uniqueness property for $e^{*}(x, t)$ in Theorem 1.3 holds. However, if one fixes $e(x, t)$ and tries to identify $a_{I}$ through problem $(P)$, then the uniqueness of $a_{I}$ is indeed guaranteed as the following theorem shows:

Theorem 1.5. Under the same notation as in Theorem 1.3, there exists a unique $a_{I}^{*} \in \mathbf{R}^{\mathcal{K}}$ such that

$$
\int_{Q^{\omega}}\left|u\left(e, a_{I}^{*} ; x, t\right)-\widetilde{u}\right|^{2} d x d t=\inf _{a_{I} \in \mathbf{R}^{\mathcal{K}}} \int_{Q^{\omega}}\left|u\left(e, a_{I} ; x, t\right)-\widetilde{u}\right|^{2} d x d t .
$$

System (1.1) models a large class of physical processes, where $u(x, t)$ represents the temperature or other physical quantity. The identification problems associated with system (1.1) with initial condition $u(x, 0)=u_{0}(x)$, where $u_{0}(x)$ is a given function, were studied by many authors. See [1] [4] [6] [7] and [13], where the observations are taken in the whole domain $\Omega$. However, in many applications, one may only be able to measure the quantity on a subdomain $\omega \subset \Omega$ and does not have enough information about the initial value. One may still be asked to determine the error influence $e(x, t)$ in the physical process through the approximate value of the solutions over $\omega$. When the approximate value comes from approximate periodic solutions, then our results of the present paper can be directly applied. Notice that this is an inverse problem. For the direct problem, one is asked to determine the value of the solution to (1.1) for a given $e(x, t)$ and $a_{I}$.

Since our observation is taken in a subdomain $\omega \subset \Omega$, we can not apply the method employed in the work mentioned above to answer $(P)$. Our key ingredients in this paper to get the existence of solutions for $(P)$ are the energy estimate (1.5) and the Carleman inequality. There have been many papers written on the related subjects in recent years. Here we would like to mention [1-7] [14] [16] [17], and the reference therein, to name a few.

The paper is organized as follows. In Section 2, we prove the existence and uniqueness of the solution to system (1.4). In Section 3, we obtain the existence of the identification problem $(P)$ by proving Theorem 1.3.

This work is a continuation of [14], where very special cases of the results in this paper were studied. It should be mentioned that the paper is largely motivated by two papers of G. Wang and L. Wang [16] [17]. 


\section{The existence and uniqueness of the solution}

In this section, we prove the existence and uniqueness of the solution to system (1.4). We will use the Galerkin method for constructing solutions in the $\mathcal{S}_{\mathcal{K}}$-space for the following equation introduced in Section 1:

$$
\begin{cases}\frac{\partial u(x, t)}{\partial t}+L_{0} u(x, t)+e(x, t) u(x, t)=f(x, t), & \text { in } Q, \\ u(x, t)=0, & \text { on } \Sigma .\end{cases}
$$

Recall that $L_{0} X_{j}(x)=\lambda_{j} X_{j}(x), \lambda_{j} \rightarrow \infty, X_{j}(x) \in H_{0}^{1}(\Omega)$. Let $G_{N}=\{g(x, t) \in$ $\left.L^{2}(Q) ; g(x, t)=\sum_{j=1}^{N} g_{j}(t) X_{j}(x), g_{j}(t) \in L^{2}(0, T)\right\}$. We first look for an approximate solution $u^{N}(x, t)$ of $(2.1)$ in the $G_{N}$-space, which also has the $\mathcal{K}$-approximate periodicity as defined before. Here $\mathcal{K}$ depends only on the $L_{0}, M, \Omega$ and will be determined later. $N$ is always assumed to be sufficiently large $(N>>\mathcal{K})$.

Write $L=L_{0}+e$. Assume $u^{N}=\sum_{j=1}^{N} u_{j}^{N}(t) X_{j}(x)$ such that $\partial_{t} u^{N}=-L u^{N}+f$ has 0 projection to $G_{N}$ in the following sense:

$$
\left(\partial_{t} u^{N}+L u^{N}-f, \varphi\right)=0, \quad \text { for } 0<t<T \text { and any } \varphi \in G_{N} .
$$

Letting $\varphi=X_{j}$ for $j=1,2, \cdots, N$, we get the following system of ordinary differential equations:

$$
\begin{aligned}
& \frac{d u_{j}^{N}(t)}{d t}+\sum B_{k j}(t) u_{k}^{N}(t)=f_{j}(t), j=1,2, \cdots, N, \\
& \text { where } B_{k j}(t)=\left(L X_{k}, X_{j}\right)=\int_{\Omega} L X_{k} \cdot X_{j} d x, f_{j}(t)=\left(f, X_{j}\right)=\int_{\Omega} f(x, t) X_{j}(x) d x .
\end{aligned}
$$

We put the following condition on $u_{j}^{N}$ :

$u_{j}^{N}(0)=u_{j}^{N}(T)$ for $j>\mathcal{K}$ with $\mathcal{K}$ independent of $N$ and being determined later.

Consider the following system of ordinary differential equations:

$$
\left\{\begin{array}{l}
\frac{d u_{j}^{N}(t)}{d t}+\sum B_{k j}(t) u_{k}^{N}(t)=0, j=1, \cdots, N \\
u_{I}^{N}(0)=0 \\
u_{I I}^{N}(0)=a_{I I}^{N} \in \mathbf{R}^{N-\mathcal{K}}
\end{array}\right.
$$

Here and in what follows,

$$
\begin{aligned}
& u_{I}^{N}(t)=\left(u_{1}^{N}(t), u_{2}^{N}(t), \cdots, u_{\mathcal{K}}^{N}(t)\right), \\
& u_{I I}^{N}(t)=\left(u_{\mathcal{K}+1}^{N}(t), u_{\mathcal{K}+2}^{N}(t), \cdots, u_{N}^{N}(t)\right) .
\end{aligned}
$$

Lemma 2.1. Let $u^{N}(x, t)=\sum_{j=1}^{N} u_{j}^{N}(t) X_{j}(x)$ be the solution of (2.2). There exists an integer $\mathcal{K}$ depending only on $L_{0}, M, \Omega$ such that for any fixed $N>\mathcal{K}$, the operator:

$$
J: \mathbf{R}^{N-\mathcal{K}} \longmapsto \mathbf{R}^{N-\mathcal{K}}, J\left(a_{I I}^{N}\right)=u_{I I}^{N}(T),
$$


is contractive. Namely,

$$
\left|J\left(a_{I I}^{N}\right)\right| \leq \mu\left|a_{I I}^{N}\right| \text { with } \mu \text { fixed and } 0 \leq \mu<1 .
$$

Here and in what follows, we always assume that ess $\sup _{t \in(0, T)}\|e(x, t)\|_{L^{q}(\Omega)} \leq M$.

For the proof of Lemma 2.1, we need the following claim:

Claim 2.2. For any $v_{1}, v_{2} \in H_{0}^{1}(\Omega)$, there are constants $C(\Omega, q)$ depending only on $\Omega, q$ and $C_{s}(\varepsilon)$ and $C_{l}(\varepsilon)$, depending only on $\varepsilon$ with $C_{s}(\varepsilon) \rightarrow 0$ as $\varepsilon \rightarrow 0, C_{l}(\varepsilon) \rightarrow \infty$ as $\varepsilon \rightarrow 0$, such that

$$
\begin{aligned}
\int_{\Omega}\left|e(x, t) v_{1}(x) v_{2}(x)\right| d x & \leq C(\Omega, q) M\left\{C_{l}(\varepsilon)\left(\left\|v_{1}\right\|_{L^{2}(\Omega)}^{2}+\left\|v_{2}\right\|_{L^{2}(\Omega)}^{2}\right)\right. \\
& \left.+C_{s}(\varepsilon)\left(\left\|\nabla v_{1}\right\|_{L^{2}(\Omega)}^{2}+\left\|\nabla v_{2}\right\|_{L^{2}(\Omega)}^{2}\right)\right\} .
\end{aligned}
$$

Proof of Claim 2.2. By the Schwartz inequality, we need only to prove the claim in the case of $v_{1}=v_{2}=v$.

$$
\begin{aligned}
\int_{\Omega}|e(x, t)| v^{2} d x & \leq\|e(\cdot, t)\|_{L^{q}(\Omega)}\left\|v^{2}\right\|_{L^{q^{\prime}}(\Omega)} \\
& \leq M\|v\|_{L^{2 q^{\prime}}(\Omega)}^{2}
\end{aligned}
$$

where $q^{\prime}=\frac{q}{q-1}$. Let $\alpha=\frac{1}{2 q^{\prime}}\left(n+2-n q^{\prime}\right)$. Then $\frac{1}{2 q^{\prime}}=\frac{\alpha}{2}+\frac{1-\alpha}{\frac{2(n+2)}{n}}$. Next, by the Hölder inequality, we have

$$
I=\int_{\Omega}|e(x, t)| v^{2} d x \leq M\|v\|_{L^{2}(\Omega)}^{2 \alpha}\|v\|_{L^{\frac{2(n+2)}{n}(\Omega)}}^{2(1-\alpha)} .
$$

By the Sobolev inequality,

$$
\|v\|_{L \frac{2(n+2)}{n}(\Omega)} \leq C(\Omega, q)\|v\|_{H_{0}^{1}(\Omega)} .
$$

Hence, $I \leq M C(\Omega, q)\left(\|v\|_{L^{2}(\Omega)}^{\alpha}\|v\|_{H_{0}^{1}(\Omega)}^{1-\alpha}\right)^{2}$.

Now, by the following Hölder inequality:

$$
a \cdot b \leq \varepsilon a^{p}+\frac{1}{\varepsilon^{\frac{p}{q}}} b^{q}
$$

with $\frac{1}{p}+\frac{1}{q}=1$, we have

$$
\begin{aligned}
I & \leq M C(\Omega, q)\left(C_{s}(\varepsilon)\|v\|_{H_{0}^{1}(\Omega)}+C_{l}(\varepsilon)\|v\|_{L^{2}(\Omega)}\right)^{2} \\
& \leq M C(\Omega, q)\left(C_{s}(\varepsilon)\|v\|_{H_{0}^{1}(\Omega)}^{2}+C_{l}(\varepsilon)\|v\|_{L^{2}(\Omega)}^{2}\right) \\
& \leq M C(\Omega, q)\left(C_{s}(\varepsilon)\|\nabla v\|_{L^{2}(\Omega)}^{2}+C_{l}(\varepsilon)\|v\|_{L^{2}(\Omega)}^{2}\right) .
\end{aligned}
$$


Here and in what follows, $C_{s}(\varepsilon), C_{l}(\varepsilon)$ stand for small and large constant depending only on $\varepsilon$, which may be different in different contexts. The proof of the claim is complete.

By Claim 2.2, we have

$$
\begin{aligned}
\left|B_{k j}(t)\right| & =\left|\left(L_{0} X_{k}, X_{j}\right)+\left(e(x, t) X_{k}, X_{j}\right)\right| \\
& =\left|\lambda_{j} \delta_{j}^{k}+\left(e(x, t) X_{k}, X_{j}\right)\right| \\
& \leq\left|\lambda_{j} \delta_{j}^{k}\right|+\left|C_{l}(\varepsilon)+C_{s}(\varepsilon)\left(\left\|\nabla X_{k}\right\|_{L^{2}(\Omega)}^{2}+\left\|\nabla X_{j}\right\|_{L^{2}(\Omega)}^{2}\right)\right|, \\
\left|f_{j}(t)\right| & \leq\left(\int_{\Omega} f^{2}(x, t) d x\right)^{\frac{1}{2}}\left(\int_{\Omega} X_{j}^{2} d x\right)^{\frac{1}{2}} \leq\|f\|_{L^{2}(\Omega)} .
\end{aligned}
$$

In particular, we conclude that any solution of the initial value problem of

$$
\left\{\begin{array}{l}
\frac{d u_{j}^{N}(t)}{d t}+\sum B_{k j}(t) u_{k}^{N}(t)=f_{j}(t), j=1, \cdots, N, \\
\left(u_{1}^{N}(0), u_{2}^{N}(0), \cdots, u_{N}^{N}(0)\right)=a^{N} \in \mathbf{R}^{N},
\end{array}\right.
$$

is absolutely continuous over $[0, T]$.

Proof of Lemma 2.1. Multiplying $2 u_{j}^{N}(t)$ to the first equation of (2.2) and summing up with respect to $\mathrm{j}$ from 1 to $N$, we get

$$
\frac{d\left(\left\|u^{N}(\cdot, t)\right\|^{2}\right)}{d t}+2\left(L_{0} u^{N}, u^{N}\right)+2\left(e(x, t) u^{N}, u^{N}\right)=0 .
$$

As before, we use $\|\cdot\|$ to denote the usual $L^{2}(\Omega)$-norm. After some calculation involving the Green formula, we have the following Gärding inequality (see [8]):

$$
\frac{2}{\lambda^{*}}\left\|\nabla u^{N}\right\|^{2}+C\left\|u^{N}\right\|^{2} \geq\left(L_{0} u^{N}, u^{N}\right) \geq \frac{\lambda^{*}}{2}\left\|\nabla u^{N}\right\|^{2}-C\left\|u^{N}\right\|^{2} .
$$

By (2.4) and Claim 2.2, we obtain

$$
\frac{d\left(\left\|u^{N}(\cdot, t)\right\|^{2}\right)}{d t}+\lambda^{*}\left\|\nabla u^{N}\right\|^{2}-C\left\|u^{N}\right\|^{2}-C_{s}(\varepsilon)\left\|\nabla u^{N}\right\|^{2}-C_{l}(\varepsilon)\left\|u^{N}\right\|^{2} \leq 0 .
$$

We choose $\varepsilon$ such that $C_{s}(\varepsilon)<\frac{\lambda^{*}}{2}$. Then we get, for a large constant $C_{l}$,

$$
\frac{d\left(\left\|u^{N}(\cdot, t)\right\|^{2}\right)}{d t}+\frac{\lambda^{*}}{2}\left\|\nabla u^{N}\right\|^{2}-C_{l}(\varepsilon)\left\|u^{N}\right\|^{2} \leq 0 .
$$

Applying the Gronwall inequality, we have

$$
\begin{gathered}
\frac{d}{d t}\left(\left\|u^{N}(\cdot, t)\right\|^{2} e^{-C_{l}(\varepsilon) t}\right)+\frac{\lambda^{*}}{2} e^{-C_{l}(\varepsilon) t}\left\|\nabla u^{N}\right\|^{2} \leq 0, \\
\left\|u^{N}(\cdot, t)\right\|^{2}+\int_{0}^{t}\left\|\nabla u^{N}(\cdot, \tau)\right\|^{2} d \tau \leq C\left\|u^{N}(\cdot, 0)\right\|^{2}, \quad \forall t \in[0, T] .
\end{gathered}
$$


In particular,

$$
\left\|u^{N}(\cdot, T)\right\|^{2} \leq C\left\|u^{N}(\cdot, 0)\right\|^{2}=C\left|a_{I I}^{N}\right|^{2}, \text { and } \int_{0}^{T}\left\|\nabla u^{N}(\cdot, t)\right\|^{2} d t \leq C\left|a_{I I}^{N}\right|^{2} .
$$

Next, multiplying $2 u_{j}^{N}(t)$ to the first equation of (2.2) and summing up with respect to $j$ from $\mathcal{K}+1$ to $N$, and letting

$$
u^{N, I I}(x, t)=\sum_{j=\mathcal{K}+1}^{N} u_{j}^{N}(t) X_{j}(x)
$$

then we get

$$
\frac{d\left(\left\|u^{N, I I}(\cdot, t)\right\|^{2}\right)}{d t}+2\left(L u^{N}, u^{N, I I}\right)=0
$$

Notice that

$$
\begin{aligned}
\left(L u^{N}, u^{N, I I}\right) & =\left(L_{0} u^{N}, u^{N, I I}\right)+\left(e(x, t) u^{N}, u^{N, I I}\right) \\
& =\left(L_{0} u^{N, I I}, u^{N, I I}\right)+\left(e(x, t) u^{N}, u^{N, I I}\right) \\
& =\sum_{j=\mathcal{K}+1}^{N} \lambda_{j}\left(u_{j}^{N}(t)\right)^{2}+\left(e(x, t) u^{N}, u^{N, I I}\right) .
\end{aligned}
$$

By Claim 2.2 and (2.4), we have

$$
\begin{aligned}
\mid\left(e(x, t) u^{N},\right. & \left.u^{N, I I}\right) \mid \leq C_{l}(\varepsilon)\left(\left\|u^{N}\right\|^{2}+\left\|u^{N, I I}\right\|^{2}\right)+C_{s}(\varepsilon)\left(\left\|\nabla u^{N}\right\|^{2}+\left\|\nabla u^{N, I I}\right\|^{2}\right) \\
& \leq C_{l}(\varepsilon)\left\|u^{N}\right\|^{2}+C_{s}(\varepsilon)\left\|\nabla u^{N}\right\|^{2}+C_{s}(\varepsilon)\left\{\frac{2 C}{\lambda^{*}}\left\|u^{N, I I}\right\|^{2}+\frac{2}{\lambda^{*}}\left(L_{0} u^{N, I I}, u^{N, I I}\right)\right\} \\
& \leq C_{l}(\varepsilon)\left\|u^{N}\right\|^{2}+C_{s}(\varepsilon)\left\|\nabla u^{N}\right\|^{2}+C_{s}(\varepsilon)\left\{\frac{2 C}{\lambda^{*}}\left\|u^{N}\right\|^{2}+\frac{2}{\lambda^{*}} \sum_{j=\mathcal{K}+1}^{N} \lambda_{j}\left(u_{j}^{N}(t)\right)^{2}\right\} \\
& \leq C_{l}(\varepsilon)\left\|u^{N}\right\|^{2}+C_{s}(\varepsilon)\left\|\nabla u^{N}\right\|^{2}+C_{s}(\varepsilon)\left\{\frac{2 C}{\lambda^{*}}\left\|u^{N}\right\|^{2}+\frac{2}{\lambda^{*}} \sum_{j=1}^{N} \lambda_{j}\left(u_{j}^{N}(t)\right)^{2}\right\} \\
& \leq C_{l}(\varepsilon)\left\|u^{N}\right\|^{2}+C_{s}(\varepsilon)\left\|\nabla u^{N}\right\|^{2}+C_{s}(\varepsilon)\left\{\frac{2 C}{\lambda^{*}}\left\|u^{N}\right\|^{2}+\frac{2}{\lambda^{*}}\left(L_{0} u^{N}, u^{N}\right)\right\} \\
& \leq C_{l}(\varepsilon)\left\|u^{N}\right\|^{2}+C_{s}(\varepsilon)\left\|\nabla u^{N}\right\|^{2}+C_{s}(\varepsilon)\left(\frac{4 C}{\lambda^{*}}\left\|u^{N}\right\|^{2}+\frac{4 C}{\lambda^{* 2}}\left\|\nabla u^{N}\right\|^{2}\right) \\
& \leq C_{l}(\varepsilon)\left\|u^{N}\right\|^{2}+C_{s}(\varepsilon)\left\|\nabla u^{N}\right\|^{2} .
\end{aligned}
$$

Notice that to get the last inequality, we applied the other part of the Gärding estimate.

We have

$$
\frac{d\left(\left\|u^{N, I I}(\cdot, t)\right\|^{2}\right)}{d t}+2 \lambda_{\mathcal{K}}\left\|u^{N, I I}\right\|^{2}-C_{l}(\varepsilon)\left\|u^{N}\right\|^{2}-C_{s}(\varepsilon)\left\|\nabla u^{N}\right\|^{2} \leq 0 .
$$

By the Gronwall inequality, we get

$$
\begin{aligned}
e^{2 \lambda_{\mathcal{K}} t}\left\|u^{N, I I}(\cdot, t)\right\|^{2}-\left\|u^{N, I I}(\cdot, 0)\right\|^{2} & \leq C_{l}(\varepsilon) \int_{0}^{t} e^{2 \lambda_{\mathcal{K}} \tau}\left\|u^{N}\right\|^{2} d \tau \\
& +C_{s}(\varepsilon) \int_{0}^{t} e^{2 \lambda_{\mathcal{K}} \tau}\left\|\nabla u^{N}\right\|^{2} d \tau, \quad \forall t \in[0, T] .
\end{aligned}
$$

We obtain

$$
\begin{aligned}
e^{2 \lambda_{\mathcal{K}} T}\left\|u^{N, I I}(\cdot, T)\right\|^{2}-\left\|u^{N, I I}(\cdot, 0)\right\|^{2} & \leq C_{l}(\varepsilon) \int_{0}^{T} e^{2 \lambda_{\mathcal{K}} t}\left\|u^{N}\right\|^{2} d t \\
& +C_{s}(\varepsilon) \int_{0}^{T} e^{2 \lambda_{\mathcal{K}} t}\left\|\nabla u^{N}\right\|^{2} d t
\end{aligned}
$$




$$
\begin{aligned}
\left\|u^{N, I I}(\cdot, T)\right\|^{2} & \leq e^{-2 \lambda_{\mathcal{K}} T}\left\|u^{N, I I}(\cdot, 0)\right\|^{2}+C_{l}(\varepsilon) \int_{0}^{T} e^{2 \lambda_{\mathcal{K}}(t-T)}\left\|u^{N}\right\|^{2} d t \\
& +C_{s}(\varepsilon) \int_{0}^{T} e^{2 \lambda_{\mathcal{K}}(t-T)}\left\|\nabla u^{N}\right\|^{2} d t \\
& \leq e^{-2 \lambda_{\mathcal{K}} T}\left|a_{I I}^{N}\right|^{2}+C_{l}(\varepsilon) \cdot C \cdot\left|a_{I I}^{N}\right|^{2}\left(\frac{1}{2 \lambda_{\mathcal{K}}}-\frac{e^{-2 \lambda_{\mathcal{K}} T}}{2 \lambda_{\mathcal{K}}}\right)+C_{s}(\varepsilon) \cdot C \cdot\left|a_{I I}^{N}\right|^{2} .
\end{aligned}
$$

Now, we first choose $\varepsilon$ sufficient small such that $C_{s}(\varepsilon) \cdot C<\frac{1}{4}$. Then we fix such an $\varepsilon$ and fix a $\mathcal{K} \gg 1$ such that $e^{-2 \lambda_{\mathcal{K}} T}<\frac{1}{4}$ and $C_{l}(\varepsilon) \cdot C \cdot\left(\frac{1}{2 \lambda_{\mathcal{K}}}-\frac{e^{-2 \lambda_{\mathcal{K}} T}}{2 \lambda_{\mathcal{K}}}\right)<\frac{1}{4}$. (Apparently, the choice of such a $\mathcal{K}$ depends only on the operator $L_{0}, M, \Omega$.) We then obtain

$$
\left|u_{I I}^{N}(T)\right|^{2}=\left\|u^{N, I I}(\cdot, T)\right\|^{2} \leq \frac{3}{4}\left|a_{I I}^{N}\right|^{2} .
$$

Since $J\left(a_{I I}^{N}\right)=u_{I I}^{N}(T)$, we see the proof of Lemma 2.1.

Next, we prove the following proposition:

Proposition 2.3. Let $\mathcal{K}$ be choose as above. Then for any $a_{I}^{N} \in \mathbf{R}^{\mathcal{K}}$, there exists a unique solution $u^{N}$ of the following mixed boundary value problem:

$$
\left\{\begin{array}{l}
\frac{d u_{j}^{N}(t)}{d t}+\sum_{k j} B_{k j}(t) u_{k}^{N}(t)=f_{j}(t), j=1, \cdots, N, \\
u_{I}^{N}(0)=a_{I}^{N} \in \mathbf{R}^{\mathcal{K}}, \\
u_{I I}^{N}(0)=u_{I I}^{N}(T) .
\end{array}\right.
$$

Proof of Proposition 2.3. Let $u_{*}^{N}(x, t)=\sum_{j=1}^{N} u_{*, j}^{N}(t) X_{j}(x)$ be the solution of the following system:

$$
\left\{\begin{array}{l}
\frac{d u_{j}^{N}(t)}{d t}+\sum B_{k j}(t) u_{k}^{N}(t)=f_{j}(t), j=1, \cdots, N, \\
\left(u_{1}^{N}(0), u_{2}^{N}(0), \cdots, u_{N}^{N}(0)\right)=0 .
\end{array}\right.
$$

Let $u_{a^{N}}^{N}(x, t)=\sum_{j=1}^{N} u_{a^{N}, j}^{N}(t) X_{j}(x)$ be the solution of the following system:

$$
\left\{\begin{array}{l}
\frac{d u_{j}^{N}(t)}{d t}+\sum B_{k j}(t) u_{k}^{N}(t)=0, j=1, \cdots, N, \\
\left(u_{1}^{N}(0), u_{2}^{N}(0), \cdots, u_{N}^{N}(0)\right)=a^{N}=\left(a_{I}^{N}, a_{I I}^{N}\right) .
\end{array}\right.
$$

For a fixed $a_{I}^{N} \in \mathbf{R}^{\mathcal{K}}$, we define

$$
\widetilde{J}_{a_{I}^{N}}: \mathbf{R}^{N-\mathcal{K}} \longrightarrow \mathbf{R}^{N-\mathcal{K}} \text { such that } \widetilde{J}_{a_{I}^{N}}\left(a_{I I}^{N}\right)=u_{* I I}^{N}(T)+u_{a^{N} I I}^{N}(T),
$$

where

$$
\begin{aligned}
& u_{* I I}^{N}(T)=\left(u_{*, \mathcal{K}+1}^{N}(T), u_{*, \mathcal{K}+2}^{N}(T), \cdots, u_{*, N}^{N}(T)\right), \\
& u_{a^{N} I I}^{N}(T)=\left(u_{a^{N}, \mathcal{K}+1}^{N}(T), u_{a^{N}, \mathcal{K}+2}^{N}(T), \cdots, u_{a^{N}, N}^{N}(T)\right) .
\end{aligned}
$$


Namely, $u_{* I I}^{N}$ is the last $N-\mathcal{K}$ components of $u_{*}^{N}$ and $u_{a^{N} I I}^{N}$ is the last $N-\mathcal{K}$ components of $u_{a^{N}}^{N}$. We have

$$
\begin{aligned}
\left|\widetilde{J}_{a_{I}^{N}}\left(a_{I I}^{N, 1}\right)-\widetilde{J}_{a_{I}^{N}}\left(a_{I I}^{N, 2}\right)\right| & =\left|u_{a^{N, 1} I I}^{N}(T)-u_{a^{N, 2} I I}^{N}(T)\right| \\
& =\left|J\left(a_{I I}^{N, 1}-a_{I I}^{N, 2}\right)\right| \\
& \leq \frac{\sqrt{3}}{2}\left|a_{I I}^{N, 1}-a_{I I}^{N, 2}\right|
\end{aligned}
$$

Hence, $\widetilde{J}_{a_{I}^{N}}$ is a contractive map and has a unique fixed point $\widetilde{a_{I I}^{N}}$. Namely, $\left.\widetilde{J}_{a_{I}^{N}} \widetilde{\left(a_{I I}^{N}\right.}\right)=\widetilde{a_{I I}^{N}}$. Then (2.7) has a solution $u^{N}(x, t)$ with $u_{I I}^{N}\left(\underset{\widetilde{J}}{0}=\widetilde{a_{I I}^{N}}=u_{I I}^{N}(T)\right.$. The uniqueness also follows from the uniqueness of the fixed point of $\widetilde{J}_{a_{I}^{N}}$. The proof of the proposition is complete.

Next, we estimate $\widetilde{a_{I I}^{N}}$, the unique fixed point of $\widetilde{J}_{a_{I}^{N}}$.

Proposition 2.4. Let $\widetilde{a_{I I}^{N}}$ be as above. Then $\left|\widetilde{a_{I I}^{N}}\right|^{2} \leq C\left(\left|a_{I}^{N}\right|^{2}+\int_{Q} f^{2} d x d t\right)$, where $C$ depends only on $L_{0}, M, \Omega$.

Proof of Proposition 2.4. We know $\widetilde{a_{I I}^{N}}=u_{I I}^{N}(T)=u_{*, I I}^{N}(T)+u_{a^{N}, I I}^{N}(T)$. We first estimate $u_{*, I I}^{N}(T)$. Multiplying the first equation of (2.8) by $2 u_{*, j}^{N}(t)$ and summing up with respect to $j$ from 1 to $N$, we have

$$
\begin{aligned}
\frac{d\left(\left\|u_{*}^{N}(\cdot, t)\right\|^{2}\right)}{d t}+2\left(L u_{*}^{N}, u_{*}^{N}\right) & =\sum_{j=1}^{N} f_{j}(t) u_{* j}^{N}(t) \\
& \leq\|f\|^{2}+\left\|u_{*}^{N}(\cdot, t)\right\|^{2} .
\end{aligned}
$$

By using the Gronwall inequality as before, we get

$$
\sup _{t \in[0, T]}\left\|u_{*}^{N}(\cdot, t)\right\|^{2}+\int_{0}^{T}\left\|\nabla u_{*}^{N}(\cdot, t)\right\|^{2} d t \leq C \int_{Q} f^{2} d x d t .
$$

In particular, $\left\|u_{*}^{N}(\cdot, T)\right\|^{2} \leq C \int_{Q} f^{2} d x d t$. Hence,

$$
\left|u_{*, I I}^{N}(T)\right|^{2}=\left\|u_{* I I}^{N}(\cdot, T)\right\|^{2} \leq C \int_{Q} f^{2} d x d t .
$$

Next, we let $u^{N, 1}(x, t), u^{N, 2}(x, t)$ be the solution of the following system (2.11) and (2.12), respectively,

$$
\begin{aligned}
& \left\{\begin{array}{l}
\frac{d u_{j}^{N}(t)}{d t}+\sum B_{k j}(t) u_{k}^{N}(t)=0, j=1, \cdots, N, \\
\left(u_{1}^{N}(0), u_{2}^{N}(0), \cdots, u_{N}^{N}(0)\right)=\left(a_{I}^{N}, 0\right),
\end{array}\right. \\
& \left\{\begin{array}{l}
\frac{d u_{j}^{N}(t)}{d t}+\sum B_{k j}(t) u_{k}^{N}(t)=0, j=1, \cdots, N, \\
\left(u_{1}^{N}(0), u_{2}^{N}(0), \cdots, u_{N}^{N}(0)\right)=\left(0, \widetilde{a_{I I}^{N}}\right) .
\end{array}\right.
\end{aligned}
$$


From the proof of Proposition 2.3 and Lemma 2.1, we get

$$
\begin{aligned}
\left|u_{a^{N} I I}^{N}(T)\right|^{2} & =\left|u_{I I}^{N, 1}(T)+u_{I I}^{N, 2}(T)\right|^{2} \\
& \leq C_{l}(\varepsilon)\left|a_{I}^{N}\right|^{2}+\left(1+C_{s}(\varepsilon)\right)\left|J\left(\widetilde{a_{I I}^{N}}\right)\right|^{2} \\
& \leq C_{l}\left|a_{I}^{N}\right|^{2}+\left(1+C_{s}\right) \frac{3}{4}\left|\widetilde{a_{I I}^{N}}\right|^{2} .
\end{aligned}
$$

By (2.10) and (2.13), we have

$$
\left|\widetilde{a_{I I}^{N}}\right|^{2} \leq C\left(\left|a_{I}^{N}\right|^{2}+\int_{Q} f^{2} d x d t\right)
$$

The proof of the proposition is complete.

Remark 2.5. From the proof of Lemma 2.1, we conclude that the value $\mathcal{K}$ depends only on $L_{0}, \Omega$ and $M$. Namely, given $L_{0}$ and $M$ with $\operatorname{ess} \sup _{t \in(0, T)}\|e(x, t)\|_{L^{q}(\Omega)} \leq M$, there is a $\mathcal{K}_{0} \equiv \mathcal{K}_{0}\left(L_{0}, M, \Omega\right)$ such that for any $N>\mathcal{K} \geq \mathcal{K}_{0}$ and $a_{I}^{N}=\left(a_{1}^{N}, a_{2}^{N}, \cdots, a_{\mathcal{K}}^{N}\right)$ $\in \mathbf{R}^{\mathcal{K}}$, the following mixed value problem has a unique absolutely continuous solution $u^{N}(x, t)=\sum_{j=1}^{N} u_{j}^{N}(t) X_{j}(x)$ over $[0, T]:$

$$
\left\{\begin{array}{l}
\frac{d u_{j}^{N}(t)}{d t}+\sum_{k j} B_{k j}(t) u_{k}^{N}(t)=f_{j}(t), j=1, \cdots, N \\
u_{I}^{N}(0)=a_{I}^{N} \\
u_{I I}^{N}(0)=u_{I I}^{N}(T) .
\end{array}\right.
$$

Moreover, write $a_{I I}^{N}=u_{I I}^{N}(0)=u_{I I}^{N}(T)$, we have the estimate:

$$
\left|a_{I I}^{N}\right|^{2} \leq C\left(\left|a_{I}^{N}\right|^{2}+\int_{Q} f^{2} d x d t\right)
$$

where $C$ depends only on $L_{0}, M, \Omega$.

Now, we follow the standard method to provide a convergence proof for the $\mathcal{K}$ approximate periodic solution $u^{N}(x, t)$ in Proposition 2.3. Since some minor changes are needed, we give some details. We first recall the energy estimate (Chapter 3 of [5]):

$$
\sup _{t \in[0, T]}\left\|u^{N}(\cdot, t)\right\|^{2}+\int_{0}^{T}\left\|\nabla u^{N}(\cdot, t)\right\|^{2} d t \leq C\left(\left\|u^{N}(\cdot, 0)\right\|^{2}+\int_{Q} f^{2} d x d t\right) .
$$

By the estimate in $(2.14)^{\prime}$, we get

$$
\sup _{t \in[0, T]}\left\|u^{N}(\cdot, t)\right\|^{2}+\int_{0}^{T}\left\|\nabla u^{N}(\cdot, t)\right\|^{2} d t \leq C\left(\left|a_{I}^{N}\right|^{2}+\int_{Q} f^{2} d x d t\right),
$$


where $C$ depends only on $L_{0}, M, \Omega$, and where $u^{N}(x, t)$ is a solution of $(2.7)$. We next fix $a_{I}^{N}=a_{I}=\left(a_{1}, a_{2}, \cdots, a_{\mathcal{K}}\right)$, and also $\mathcal{K}$ that satisfies the property in Remark 2.5. We compute

$$
\begin{aligned}
\left|u_{j}^{N}(t+\triangle t)-u_{j}^{N}(t)\right| & =\left|\int_{t}^{t+\triangle t}\left(\sum B_{k j}(\tau) u_{k}^{N}(\tau)-f_{j}(\tau)\right) d \tau\right| \\
& \leq\left|\int_{t}^{t+\triangle t}\left(L u^{N}, X_{j}\right) d \tau\right|+\left|\int_{t}^{t+\triangle t} f_{j}(\tau) d \tau\right| \\
& \leq\left|\int_{t}^{t+\triangle t}\left\{C_{l}(\varepsilon)\left\|\nabla X_{j}\right\|^{2}+C_{s}(\varepsilon)\left\|\nabla X_{j}\right\|^{2}+C_{s}(\varepsilon)\left\|\nabla u^{N}\right\|^{2}+C\right\} d \tau\right| \\
& +\left(\int_{t}^{t+\triangle t} 1 \cdot d \tau\right)^{\frac{1}{2}}\left(\int_{t}^{t+\triangle t} f_{j}^{2}(\tau) d \tau\right)^{\frac{1}{2}} \\
& \leq C_{s}(\varepsilon)+C_{l}(\varepsilon) \sqrt{\triangle t} .
\end{aligned}
$$

Now for any $\varepsilon^{\prime}>0$, we can find $\varepsilon>0$ such that $C_{s}(\varepsilon)<\frac{\varepsilon^{\prime}}{2}$. Then fixing such an $\varepsilon$, we can find $\delta>0$ such that when $|\triangle t|<\delta, C_{l}(\varepsilon) \sqrt{\triangle t}<\frac{\varepsilon^{\prime}}{2}$. Hence, when $|\triangle t|<\delta$,

$$
\left|u_{j}^{N}(t+\triangle t)-u_{j}^{N}(t)\right|<\varepsilon^{\prime} .
$$

Note that $\delta$ is independent of $N$. We proved that $\left\{u_{j}^{N}(t)\right\}_{N=1}^{\infty}$ is equi-continuous. Since for any $N>>1, \sum_{j=1}^{N}\left|u_{j}^{N}(t)\right|^{2} \leq\left\|u^{N}(\cdot, t)\right\|^{2} \leq C,\left\{u_{j}^{N}(t)\right\}_{N=1}^{\infty}$ is uniformly bounded. Now by the Ascoli-Arzela Theorem and the diagonal-element picking method, we can find a subsequence $\left\{N_{l}\right\}$ such that for each $j, u_{j}^{N_{l}}(t) \rightarrow u_{j}(t)$ uniformly over $[0, T]$.

Remark 2.6. We note that for each $j,\left\{u_{j}^{N}(t)\right\}_{N=1}^{\infty}$ is an equi-continuous family and uniformly bounded. For each $j$ and for any $\varepsilon>0$, there is a $\delta(j, \varepsilon)$ with $\delta$ depending only on $j, L_{0}, M, \varepsilon$ such that when $|\triangle t|<\delta(j, \varepsilon)$, we have $\left|u_{j}(t+\triangle t)-u_{j}(t)\right|<\varepsilon$.

Next, for any fixed $m<N_{l}$, we have

$$
\sum_{j=1}^{m}\left(u_{j}^{N_{l}}(t)\right)^{2} \leq \sum_{j=1}^{N_{l}}\left(u_{j}^{N_{l}}(t)\right)^{2}=\left\|u^{N_{l}}(\cdot, t)\right\|^{2} \leq C .
$$

Letting $N_{l} \rightarrow \infty$, we get $\sum_{j=1}^{m}\left(u_{j}(t)\right)^{2} \leq C$, and thus $\sum_{j=1}^{\infty}\left(u_{j}(t)\right)^{2} \leq C$. Let $u(x, t)=$ $\sum_{j=1}^{\infty} u_{j}(t) X_{j}(x)$. We have $u(x, t) \in L^{\infty}\left(0, T ; L^{2}(\Omega)\right)$. Now, for $j \leq \mathcal{K}$,

$$
u_{j}(0)=\lim _{N_{l} \rightarrow \infty} u_{j}^{N_{l}}(0)=\lim _{N_{l} \rightarrow \infty} a_{j}=a_{j}
$$

For $j>\mathcal{K}$,

$$
u_{j}(0)=\lim _{N_{l} \rightarrow \infty} u_{j}^{N_{l}}(0)=\lim _{N_{l} \rightarrow \infty} u_{j}^{N_{l}}(T)=u_{j}(T) .
$$

Since $u^{N_{l}}(x, t), \nabla u^{N_{l}}(x, t) \in L^{2}(Q)$ with $\left\|u^{N_{l}}(x, t)\right\|_{L^{2}(Q)} \leq C,\left\|\nabla u^{N_{l}}(x, t)\right\|_{L^{2}(Q)} \leq C$, without loss of generality, we can assume that $u^{N_{l}}(x, t) \rightarrow u^{*}(x, t)$ weakly in $L^{2}\left(0, T ; H_{0}^{1}(\Omega)\right)$. 
(See page 54 of [5]).

Let $\theta_{j}(t) \in C^{\infty}[0, T]$, and $\Phi^{r}(x, t)=\sum_{j=1}^{r} \theta_{j}(t) X_{j}(x), N_{l}>r$. Since

$$
\begin{aligned}
& \int_{0}^{T}\left(u^{*}(\cdot, t), \Phi^{r}(\cdot, t)\right) d t=\lim _{N_{l} \rightarrow \infty} \int_{0}^{T}\left(u^{N_{l}}(\cdot, t), \Phi^{r}(\cdot, t)\right) d t \\
& =\lim _{N_{l} \rightarrow \infty} \int_{0}^{T} \sum_{j=1}^{r} u_{j}^{N_{l}}(t) \theta_{j}(t) d t=\int_{0}^{T} \sum_{j=1}^{r} u_{j}(t) \theta_{j}(t) d t \\
& =\int_{0}^{T}\left(u(\cdot, t), \Phi^{r}(\cdot, t)\right) d t .
\end{aligned}
$$

Since the set of all such $\Phi^{r}(x, t)^{\prime} s$ is dense in $L^{2}\left(0, T ; H_{0}^{1}(\Omega)\right)$, we get $u(x, t) \equiv u^{*}(x, t)$ a.e. in $L^{2}\left(0, T ; H_{0}^{1}(\Omega)\right)$. Hence, $u(x, t) \in L^{\infty}\left(0, T ; L^{2}(\Omega)\right) \cap L^{2}\left(0, T ; H_{0}^{1}(\Omega)\right)$.

Now we prove $u(x, t)$ is a weak solution.

Let $\Phi^{r}(x, t)=\sum_{j=1}^{r} \theta_{j}(t) X_{j}(x)$ be as above and $N_{l}>r$. We have

$$
\begin{gathered}
\lim _{N_{l} \rightarrow \infty}\left(u^{N_{l}}(\cdot, t), \Phi^{r}(\cdot, t)\right)=\lim _{N_{l} \rightarrow \infty} \sum_{j=1}^{r} u_{j}^{N_{l}}(t) \theta_{j}(t)=\sum_{j=1}^{r} u_{j}(t) \theta_{j}(t)=\left(u(\cdot, t), \Phi^{r}(\cdot, t)\right) \\
\lim _{N_{l} \rightarrow \infty} \int_{0}^{t}\left(u^{N_{l}}(\cdot, \tau), \Phi_{\tau}^{r}(\cdot, \tau)\right) d \tau=\int_{0}^{t} \lim _{N_{l} \rightarrow \infty} \sum_{j=1}^{r} u_{j}^{N_{l}}(\tau) \theta_{j}^{\prime}(\tau) d \tau \\
=\int_{0}^{t} \sum_{j=1}^{r} u_{j}(\tau) \theta_{j}^{\prime}(\tau) d \tau=\int_{0}^{t}\left(u(\cdot, \tau), \Phi_{\tau}^{r}(\cdot, \tau)\right) d \tau \\
\lim _{N_{l} \rightarrow \infty} \int_{0}^{t}\left(L u^{N_{l}}(\cdot, \tau), \Phi^{r}(\cdot, \tau)\right) d \tau=\int_{0}^{t}\left(L u(\cdot, \tau), \Phi^{r}(\cdot, \tau)\right) d \tau
\end{gathered}
$$

Therefore, we get

$$
\begin{gathered}
\left(u(\cdot, t), \Phi^{r}(\cdot, t)\right)-\int_{0}^{t}\left(u(\cdot, \tau), \Phi_{\tau}^{r}(\cdot, \tau)\right) d \tau+\int_{0}^{t}\left(L u(\cdot, \tau), \Phi^{r}(\cdot, \tau)\right) d \tau \\
=\left(u(\cdot, 0), \Phi^{r}(\cdot, 0)\right)+\int_{0}^{t}\left(f(\cdot, \tau), \Phi^{r}(\cdot, \tau)\right) d \tau
\end{gathered}
$$

Since all such $\Phi^{r}(x, t)^{\prime} s$ are dense in $H_{0}^{1,1}(Q)$, we proved that $u(x, t)$ is a weak solution of (2.1). Note that $u(x, t) \in \mathcal{S}_{\mathcal{K}}$. Summarizing the above, we proved the following:

Theorem 2.7. There exists an integer $\mathcal{K}_{0} \equiv \mathcal{K}_{0}\left(L_{0}, M, \Omega\right) \geq 0$ such that for any $\mathcal{K} \geq \mathcal{K}_{0}$ and any initial value $a_{I}=\left(a_{1}, a_{2}, \cdots, a_{\mathcal{K}}\right) \in \mathbf{R}^{\mathcal{K}}$, there is a unique solution to the following equation:

$$
\begin{cases}\frac{\partial u(x, t)}{\partial t}+L_{0} u(x, t)+e(x, t) u(x, t)=f(x, t), & \text { in } Q \\ u(x, t)=0, & \text { on } \Sigma, \\ \left(u(\cdot, 0), X_{j}\right)=a_{j}, & j \leq \mathcal{K}, \\ \left(u(\cdot, 0), X_{j}\right)=\left(u(\cdot, T), X_{j}\right), & j>\mathcal{K} .\end{cases}
$$


Theorem 2.8. Let $u(x, t)$ be as in Theorem 2.7. Then there is a constant $C$ depending only on $L_{0}, M, \Omega$ such that

$$
\sup _{t \in[0, T]}\|u(\cdot, t)\|^{2}+\int_{0}^{T}\|\nabla u(\cdot, t)\|^{2} d t \leq C\left(\left|a_{I}\right|^{2}+\int_{Q} f^{2} d x d t\right) .
$$

Proof of Theorem 2.8. By (2.15), we have

$$
\sup _{t \in[0, T]}\left\|u^{N_{l}}(\cdot, t)\right\|^{2}+\int_{0}^{T}\left\|\nabla u^{N_{l}}(\cdot, t)\right\|^{2} d t \leq C\left(\left|a_{I}\right|^{2}+\int_{Q} f^{2} d x d t\right) .
$$

Since $u^{N_{l}}(x, t) \rightarrow u^{*}(x, t)$ weakly in $L^{2}\left(0, T ; H_{0}^{1}(\Omega)\right)$, we have

$$
\begin{gathered}
\int_{0}^{T}\|\nabla u\|^{2} d t \leq \lim _{N_{l} \rightarrow \infty} \int_{0}^{T}\left\|\nabla u^{N_{l}}\right\|^{2} d t \leq C\left(\left|a_{I}\right|^{2}+\int_{Q} f^{2} d x d t\right) . \\
\left\|u^{N_{l}}(\cdot, t)\right\|^{2}=\sum_{j=1}^{N_{l}}\left(u_{j}^{N_{l}}(t)\right)^{2} \leq C\left(\left|a_{I}\right|^{2}+\int_{Q} f^{2} d x d t\right) .
\end{gathered}
$$

For any $m<N_{l}$, we have

$$
\sum_{j=1}^{m}\left(u_{j}^{N_{l}}(t)\right)^{2} \leq C\left(\left|a_{I}\right|^{2}+\int_{Q} f^{2} d x d t\right) .
$$

Letting $N_{l} \rightarrow \infty$, we get $\sum_{j=1}^{m}\left(u_{j}(t)\right)^{2} \leq C\left(\left|a_{I}\right|^{2}+\int_{Q} f^{2} d x d t\right)$. Hence,

$$
\sum_{j=1}^{\infty}\left(u_{j}(t)\right)^{2}=\|u(\cdot, t)\|^{2} \leq C\left(\left|a_{I}\right|^{2}+\int_{Q} f^{2} d x d t\right) .
$$

By (2.18) and (2.19), we have (2.17). The proof is complete.

Proof of Theorem 2.7. It suffices to prove the uniqueness part of Theorem 2.7. Indeed, we need only to show that the only solution $u(x, t)$ of (2.16) with $f=0, a_{I}=0$ is 0 . For this purpose, we first recall the energy estimate:

$$
\sup _{t \in[0, T]}\|u(\cdot, t)\|^{2}+\int_{0}^{T}\|\nabla u(\cdot, t)\|^{2} d t \leq C\left|a_{I I}\right|^{2},
$$

where

$$
a_{I I}=\left(\left(u(\cdot, 0), X_{\mathcal{K}+1}\right),\left(u(\cdot, 0), X_{\mathcal{K}+2}\right), \cdots \cdots\right) .
$$


Multiplying $X_{j}(x)$ to both sides of the first equation of (2.16) and then integrating over $\Omega$, we get

$$
\frac{d u_{j}(t)}{d t}+\left(L u, X_{j}\right)=0
$$

Write $u^{m}(x, t)=\sum_{j=\mathcal{K}+1}^{m}\left(u, X_{j}\right) X_{j}(x)=\sum_{j=\mathcal{K}+1}^{m} u_{j}(t) X_{j}(x), m>\mathcal{K}+1$. Then $u^{m}(x, 0)=$ $u^{m}(x, T)$.

Multiplying $2 u_{j}(t)$ to $(2.21)$ and summing up with respect to $j$ from $\mathcal{K}+1$ to $m$, we get

$$
\frac{d\left(\left\|u^{m}(\cdot, t)\right\|^{2}\right)}{d t}+2\left(L_{0} u, u^{m}\right)+2\left(e(x, t) u, u^{m}\right)=0 .
$$

By the same arguments as those in the proof of Lemma 2.1, we have

$$
\frac{\left(d\left\|u^{m}(\cdot, t)\right\|^{2}\right)}{d t}+2 \lambda_{\mathcal{K}}\left\|u^{m}(\cdot, t)\right\|^{2} \leq C_{s}(\varepsilon)\|\nabla u\|^{2}+C_{l}(\varepsilon)\|u\|^{2} .
$$

Using the Gronwall inequality and noticing that $u^{m}(x, 0)=u^{m}(x, T)$, we get

$$
\begin{aligned}
\left(e^{2 \lambda_{\mathcal{K}} T}-1\right)\left\|u^{m}(\cdot, 0)\right\|^{2} & \leq C_{s}(\varepsilon) \int_{0}^{T} e^{2 \lambda_{\mathcal{K}} t}\|\nabla u\|^{2} d t+C_{l}(\varepsilon) \int_{0}^{T} e^{2 \lambda_{\mathcal{K}} t}\|u\|^{2} d t \\
& \leq C_{s}(\varepsilon) e^{2 \lambda_{\mathcal{K}} T}\left|a_{I I}\right|^{2}+C_{l}(\varepsilon) \frac{\left(e^{\left.2 \lambda_{\mathcal{K}} \mathcal{T}^{T}-1\right)}\right.}{2 \lambda_{\mathcal{K}}}\left|a_{I I}\right|^{2} .
\end{aligned}
$$

So

$$
\left\|u^{m}(\cdot, 0)\right\|^{2} \leq C_{s}(\varepsilon) \frac{e^{2 \lambda} \mathcal{K}^{T}}{e^{2 \lambda} \mathcal{K}^{T}-1}\left|a_{I I}\right|^{2}+C_{l}(\varepsilon) \frac{1}{2 \lambda_{\mathcal{K}}}\left|a_{I I}\right|^{2} .
$$

Letting $m \rightarrow \infty$, we obtain

$$
\lim _{m \rightarrow \infty}\left\|u^{m}(\cdot, 0)\right\|^{2}=\lim _{m \rightarrow \infty} \sum_{j=\mathcal{K}+1}^{m}\left(u_{j}(0)\right)^{2}=\left|a_{I I}\right|^{2} \leq C_{s}(\varepsilon)\left|a_{I I}\right|^{2}+C_{l}(\varepsilon) \frac{1}{2 \lambda_{\mathcal{K}}}\left|a_{I I}\right|^{2} .
$$

We first choose $\varepsilon$ such that $C_{s}(\varepsilon)<\frac{1}{4}$, then we choose $\mathcal{K} \geq \mathcal{K}_{0} \gg 1$ such that $C_{l}(\varepsilon) \frac{1}{2 \lambda_{\mathcal{K}}}<\frac{1}{4}$. We have

$$
\left|a_{I I}\right|^{2} \leq \frac{1}{4}\left|a_{I I}\right|^{2}+\frac{1}{4}\left|a_{I I}\right|^{2} .
$$

It says $\left|a_{I I}\right|^{2} \equiv 0$. By (2.20), we apparently get $u(x, t) \equiv 0$. The proof is complete.

Proof of Theorem 1.2. Theorem 1.2 follows directly from Theorem 2.7-2.8.

\section{$3 \quad$ Existence of the solution to $(P)$}

In this section, we give a proof of Theorem 1.3. Besides results established in $\S 2$, another main ingredient to be used here is the Carleman inequality for linear parabolic equation developed in [2] [10] and [16], which in particular implies the unique continuation property for the solutions. 
First, by Theorem 2.7, there exists an integer $\mathcal{K}_{0} \equiv \mathcal{K}_{0}\left(L_{0}, M, \Omega\right) \geq 0$ such that for any $\mathcal{K} \geq \mathcal{K}_{0}$ and any initial value $a_{I}=\left(a_{1}, a_{2}, \cdots, a_{\mathcal{K}}\right) \in \mathbf{R}^{\mathcal{K}}$, we have a unique solution $u(x, t)$ to the following equation:

$$
\begin{cases}\frac{\partial u(x, t)}{\partial t}+L_{0} u(x, t)+e(x, t) u(x, t)=f(x, t), & \text { in } Q, \\ u(x, t)=0, & \text { on } \Sigma, \\ \left(u(\cdot, 0), X_{j}\right)=a_{j}, & j \leq \mathcal{K}, \\ \left(u(\cdot, 0), X_{j}\right)=\left(u(\cdot, T), X_{j}\right), & j>\mathcal{K} .\end{cases}
$$

For the proof of Theorem 1.3, we need the following Lemma:

Lemma 3.1. Let $e_{m} \in \mathcal{M}_{q}, a_{I}^{m}=\left(a_{1}^{m}, a_{2}^{m}, \cdots, a_{\mathcal{K}}^{m}\right) \in \mathbf{R}^{\mathcal{K}}$ with $m=1,2, \cdots$. And let $u_{m}(m=1,2, \cdots)$ be the solution of the following:

$$
\begin{cases}\frac{\partial u_{m}(x, t)}{\partial t}+L_{0} u_{m}(x, t)+e_{m}(x, t) u_{m}(x, t)=f_{m}(x, t), & \text { in } Q, \\ u_{m}(x, t)=0, & \text { on } \Sigma, \\ \left(u_{m}(\cdot, 0), X_{j}\right)=a_{j}^{m}, & j \leq \mathcal{K}, \\ \left(u_{m}(\cdot, 0), X_{j}\right)=\left(u_{m}(\cdot, T), X_{j}\right), & j>\mathcal{K} .\end{cases}
$$

Assume that $\left|a_{I}^{m}\right| \leq M_{0}$ with $M_{0}$ independent of the choice of $m$. Suppose that $e_{m} \rightarrow$ $e^{*} \in \mathcal{M}_{q}$ in the weak-star topology of $L^{\infty}\left(0, T, L^{q}(\Omega)\right), a_{j}^{m} \rightarrow a_{j}^{*}$ for $j=1,2, \cdots, \mathcal{K}$, and $f_{m} \rightarrow f^{*}$ in the $L^{2}(Q)$-norm. Then there is a subsequence $\left\{m_{k}\right\}$ such that $\left\{u_{m_{k}}\right\}$ converges in the weak $L^{2}$-topology to $u^{*} \in C\left([0, T] ; L^{2}(\Omega)\right) \cap L^{2}\left(0, T ; H_{0}^{1}(\Omega)\right)$ with

$$
\begin{cases}\frac{\partial u^{*}(x, t)}{\partial t}+L_{0} u^{*}(x, t)+e^{*}(x, t) u^{*}(x, t)=f^{*}(x, t), & \text { in } Q, \\ u^{*}(x, t)=0, & \text { on } \Sigma, \\ \left(u^{*}(\cdot, 0), X_{j}\right)=a_{j}^{*}, & j \leq \mathcal{K} \\ \left(u^{*}(\cdot, 0), X_{j}\right)=\left(u^{*}(\cdot, T), X_{j}\right), & j>\mathcal{K} .\end{cases}
$$

Moreover, write $u_{m_{k}}(x, t)=\sum_{j=1}^{\infty} u_{j}^{m_{k}}(t) X_{j}(x)$ and $u^{*}(x, t)=\sum_{j=1}^{\infty} u_{j}^{*}(t) X_{j}(x)$. Then by choosing $m_{k}$ suitably, we have

$$
\lim _{k \rightarrow \infty} u_{j}^{m_{k}}(t)=u_{j}^{*}(t), \text { for any } t \in[0, T], j,
$$

and for any $\delta>0, u_{m_{k}}(x, t) \rightarrow u^{*}(x, t)$ strongly in the $L^{2}([\delta, T] \times \Omega)$-norm.

Proof of Lemma 3.1. By the energy estimate in Theorem 2.7, we have

$$
\sup _{t \in[0, T]}\left\|u_{m}(\cdot, t)\right\|^{2}+\int_{0}^{T}\left\|\nabla u_{m}(\cdot, t)\right\|^{2} d t \leq C\left(\left|a_{I}^{m}\right|^{2}+\int_{Q} f_{m}^{2} d x d t\right) \leq C,
$$

where $a_{I}^{m}=\left(a_{1}^{m}, a_{2}^{m}, \cdots, a_{\mathcal{K}}^{m}\right), C$ depends only on $L_{0}, M$, and $\Omega$. By the assumption in Lemma 3.1, without loss of generality, we can assume $u_{m}(x, t) \rightarrow u^{*}(x, t), \nabla u_{m}(x, t) \rightarrow$ 
$\nabla u^{*}(x, t)$ in the weak $L^{2}(Q)$-topology. Apparently, $u^{*} \in L^{2}(Q) \cap L^{2}\left(0, T ; H_{0}^{1}(\Omega)\right)$.

By Remark 2.6, $\left\{u_{j}^{m}(t)\right\}_{m=1}^{\infty}$ is an equi-continuous family and uniformly bounded for each $j$. By the diagonal-element picking method and the Ascoli-Arzela Theorem, we can find a subsequence $\left\{m_{k}\right\}$ such that

$$
\text { for each } j, u_{j}^{m_{k}}(t) \rightarrow \widetilde{u_{j}^{*}}(t) \text { uniformly over }[0, T] \text {. }
$$

Now, we let $\widetilde{u^{*}}(x, t)=\sum_{j=1}^{\infty} \widetilde{u_{j}^{*}}(t) X_{j}(x)$. Apparently, from the estimate:

$$
\sum_{j=1}^{N}\left(u_{j}^{m_{k}}(t)\right)^{2} \leq C \text { for any } N \text {, it follows that } \sum_{j=1}^{\infty}\left(\widetilde{u_{j}^{*}}(t)\right)^{2}=\left\|\widetilde{u^{*}}(\cdot, t)\right\|^{2} \leq C .
$$

Now for any $\theta_{j}(t) \in C^{\infty}[0, T]$, we have

$$
\begin{aligned}
& \lim _{k \rightarrow \infty} \int_{0}^{T}\left(u_{m_{k}}(\cdot, t), X_{j}\right) \theta_{j}(t) d t=\lim _{k \rightarrow \infty} \int_{0}^{T} u_{j}^{m_{k}}(t) \theta_{j}(t) d t \\
& =\int_{0}^{T} \widetilde{u_{j}^{*}}(t) \theta_{j}(t) d t=\int_{0}^{T}\left(\widetilde{u^{*}}(\cdot, t), X_{j}\right) \theta_{j}(t) d t .
\end{aligned}
$$

On the other hand,

$$
\lim _{k \rightarrow \infty} \int_{0}^{T}\left(u_{m_{k}}(\cdot, t), X_{j}\right) \theta_{j}(t) d t=\int_{0}^{T}\left(u^{*}(\cdot, t), X_{j}\right) \theta_{j}(t) d t .
$$

Therefore,

$$
\begin{aligned}
\int_{0}^{T}\left(u^{*}(\cdot, t)-\widetilde{u^{*}}(\cdot, t), X_{j} \theta_{j}(t)\right) d t=0, \quad 0 & =\int_{0}^{T}\left(u^{*}(\cdot, t)-\widetilde{u^{*}}(\cdot, t), \sum_{j=1}^{r} X_{j}\right) \theta_{j}(t) d t \\
& =\int_{Q}\left(u^{*}(x, t)-\widetilde{u^{*}}(x, t)\right)\left(\sum_{j=1}^{r} X_{j}(x) \theta_{j}(t)\right) d x d t \\
& =\int_{Q}\left(u^{*}(x, t)-\widetilde{u^{*}}(x, t)\right) \Phi^{r}(x, t) d x d t .
\end{aligned}
$$

Since the set of functions with the form $\Phi^{r}(x, t)=\sum_{j=1}^{r} \theta_{j}(t) X_{j}(x)$ is dense in $L^{2}\left(0, T ; H_{0}^{1}(\Omega)\right)$, we conclude that $\widetilde{u^{*}}(x, t)=u^{*}(x, t)$ a.e. in $L^{2}\left(0, T ; H_{0}^{1}(\Omega)\right)$. Therefore, $u^{*}(x, t) \in$ $L^{\infty}\left(0, T ; L^{2}(\Omega)\right) \cap L^{2}\left(0, T ; H_{0}^{1}(\Omega)\right)$.

Next, for the $\Phi^{r}(x, t)$ defined above, by the assumption,

$$
\begin{aligned}
& \left(u_{m}(\cdot, t), \Phi^{r}(\cdot, t)\right)-\int_{0}^{t}\left(u_{m}(\cdot, \tau), \Phi_{\tau}^{r}(\cdot, \tau)\right) d \tau+\int_{0}^{t}\left(L_{0} u_{m}(\cdot, \tau), \Phi^{r}(\cdot, \tau)\right) d \tau \\
+ & \int_{0}^{t}\left(e_{m} u_{m}(\cdot, \tau), \Phi^{r}(\cdot, \tau)\right) d \tau=\left(u_{m}(\cdot, 0), \Phi^{r}(\cdot, 0)\right)+\int_{0}^{t}\left(f_{m}(\cdot, \tau), \Phi^{r}(\cdot, \tau)\right) d \tau .
\end{aligned}
$$

Now, as in Section 2, to show that $u^{*}(x, t)$ is the weak solution of (3.3), it suffices to show that

$$
\lim _{k \rightarrow \infty} \int_{0}^{T} \int_{\Omega} e_{m_{k}}(x, \tau) u_{m_{k}}(x, \tau) \Phi^{r}(x, \tau) d x d \tau=\int_{0}^{T} \int_{\Omega} e^{*}(x, \tau) u^{*}(x, \tau) \Phi^{r}(x, \tau) d x d \tau
$$


for a certain subsequence $\left\{m_{k}\right\}$. Notice that

for $j \leq \mathcal{K}$,

$$
\left(u^{*}(\cdot, 0), X_{j}\right)=u_{j}^{*}(0)=\lim _{k \rightarrow \infty} u_{j}^{m_{k}}(0)=\lim _{k \rightarrow \infty} a_{j}^{m_{k}}=a_{j}^{*}
$$

for $j>\mathcal{K}$,

$$
\left(u^{*}(\cdot, 0), X_{j}\right)=\lim _{k \rightarrow \infty}\left(u_{m_{k}}(\cdot, 0), X_{j}\right)=\lim _{k \rightarrow \infty}\left(u_{m_{k}}(\cdot, T), X_{j}\right)=\left(u^{*}(\cdot, T), X_{j}\right) .
$$

Hence, the proof of Lemma 3.1 will be complete if we can prove (3.4).

Next, notice that

$$
\begin{aligned}
\left|\int_{Q} e_{m_{k}} u_{m_{k}} \Phi^{r} d x d t-\int_{Q} e^{*} u^{*} \Phi^{r} d x d t\right| & \leq\left|\int_{Q}\left(e_{m_{k}}-e^{*}\right) u^{*} \Phi^{r} d x d t\right| \\
& +\left|\int_{Q} e_{m_{k}}\left(u_{m_{k}}-u^{*}\right) \Phi^{r} d x d t\right| .
\end{aligned}
$$

Apparently, $\left|\int_{Q}\left(e_{m_{k}}-e^{*}\right) u^{*} \Phi^{r} d x d t\right| \rightarrow 0$. Therefore, it suffices to prove the following claim to complete the proof of Lemma 3.1.

Claim 3.2. There is a subsequence $\left\{m_{k}\right\}$ such that $\left|\int_{Q} e_{m_{k}}\left(u_{m_{k}}-u^{*}\right) \Phi^{r} d x d t\right| \rightarrow 0$, and for any $\delta>0$,

$$
u_{m_{k}}(x, t) \rightarrow u^{*}(x, t) \text { strongly in the } L^{2}([\delta, T] \times \Omega) \text {, as } k \rightarrow \infty .
$$

Proof of Claim 3.2. Notice that

$$
\begin{cases}\frac{\partial\left(t^{\frac{2}{3}} u_{m}(x, t)\right)}{\partial t}+L\left(t^{\frac{2}{3}} u_{m}(x, t)\right)=t^{\frac{2}{3}} f_{m}(x, t)+\frac{2}{3} t^{-\frac{1}{3}} u_{m}(x, t), & \text { in } \quad Q=\Omega \times(0, T), \\ t^{\frac{2}{3}} u_{m}(x, t)=0, & \text { on } \Sigma=\partial \Omega \times(0, T), \\ \left.t^{\frac{2}{3}} u_{m}(x, t)\right|_{t=0}=0, & \text { in } \Omega .\end{cases}
$$

By the high order energy estimates for parabolic equations (page 59, Theorem 4.1 of [5]), we have

$$
\begin{aligned}
\sup _{t \in(0, T)}\left\|\nabla\left(t^{\frac{2}{3}} u_{m}(\cdot, t)\right)\right\|^{2}+\int_{Q}\left|\partial_{t}\left(t^{\frac{2}{3}} u_{m}(x, t)\right)\right|^{2} d x d t & \leq \int_{Q}\left|t^{\frac{2}{3}} f_{m}(x, t)+\frac{2}{3} t^{-\frac{1}{3}} u_{m}(x, t)\right|^{2} d x d t \\
& \leq C .
\end{aligned}
$$

Then $\left\|t^{\frac{2}{3}} u_{m}(x, t)\right\|_{W^{1,1}(Q)} \leq C$ for all $m$. By the Rellich lemma, there is a subsequence $\left\{m_{k}\right\}$ such that $t^{\frac{2}{3}} u_{m_{k}}(x, t) \rightarrow \bar{u}(x, t)$ strongly in $L^{2}(Q)$. Next,

$$
\begin{aligned}
& \int_{Q} \bar{u}(x, t) \Phi^{r}(x, t) d x d t=\lim _{k \rightarrow \infty} \int_{Q} t^{\frac{2}{3}} u_{m_{k}}(x, t) \Phi^{r}(x, t) d x d t \\
& =\lim _{k \rightarrow \infty} \int_{Q} u_{m_{k}}(x, t) t^{\frac{2}{3}} \Phi^{r}(x, t) d x d t=\int_{Q} t^{\frac{2}{3}} u^{*}(x, t) \Phi^{r}(x, t) d x d t .
\end{aligned}
$$


Since all such $\Phi^{r}(x, t)^{\prime} s$ are dense in $L^{2}\left(0, T ; H_{0}^{1}(\Omega)\right), \bar{u}(x, t)=t^{\frac{2}{3}} u^{*}(x, t)$ a.e. in $L^{2}\left(0, T ; H_{0}^{1}(\Omega)\right)$.

Now,

$$
\begin{aligned}
& \int_{\delta}^{T} \int_{\Omega}\left|u_{m_{k}}-u^{*}\right|^{2} d x d t=\int_{\delta}^{T} \int_{\Omega} t^{-4 / 3}\left|t^{\frac{2}{3}}\left(u_{m_{k}}-u^{*}\right)\right|^{2} d x d t \\
& \leq C(\delta) \int_{\delta}^{T} \int_{\Omega}\left|t^{\frac{2}{3}}\left(u_{m_{k}}-u^{*}\right)\right|^{2} d x d t \leq C(\delta) \int_{0}^{T} \int_{\Omega}\left|t^{\frac{2}{3}}\left(u_{m_{k}}-u^{*}\right)\right|^{2} d x d t \rightarrow 0 .
\end{aligned}
$$

We thus see, for any $\delta>0$, that

$$
u_{m_{k}}(x, t) \rightarrow u^{*}(x, t) \text { strongly in the } L^{2}([\delta, T] \times \Omega) .
$$

Next, by Claim 2.2,

$$
\begin{aligned}
\left|\int_{\Omega} e_{m_{k}}\left(u_{m_{k}}-u^{*}\right) \Phi^{r} d x\right| & \leq C_{s}(\varepsilon) \int_{\Omega}\left|e_{m_{k}}\left(u_{m_{k}}-u^{*}\right)^{2}\right| d x+C_{l}(\varepsilon) \int_{\Omega}\left|e_{m_{k}}\left(\Phi^{r}\right)^{2}\right| d x \\
& \leq C_{s}(\varepsilon)\left(\int_{\Omega}\left|\nabla\left(u_{m_{k}}-u^{*}\right)\right|^{2} d x+\int_{\Omega}\left|\left(u_{m_{k}}-u^{*}\right)\right|^{2} d x\right)+C_{l}(\varepsilon) \\
& \leq C_{s}(\varepsilon) \int_{\Omega}\left|\nabla\left(u_{m_{k}}-u^{*}\right)\right|^{2} d x+C_{l}(\varepsilon) .
\end{aligned}
$$

Next,

$$
\begin{aligned}
\left|\int_{0}^{\delta} \int_{\Omega} e_{m_{k}}\left(u_{m_{k}}-u^{*}\right) \Phi^{r} d x d t\right| & \leq \int_{0}^{\delta}\left(C_{s}(\varepsilon) \int_{\Omega}\left|\nabla\left(u_{m_{k}}-u^{*}\right)\right|^{2} d x+C_{l}(\varepsilon)\right) d t \\
& \leq C_{s}(\varepsilon)+\delta C_{l}(\varepsilon) .
\end{aligned}
$$

Now, for any $\varepsilon^{\prime}>0, \exists \varepsilon\left(\varepsilon^{\prime}\right)$ such that $C_{s}(\varepsilon)<\frac{\varepsilon^{\prime}}{4}$. Then there is a $\delta=\delta\left(\varepsilon, \varepsilon^{\prime}\right)$ such that $\delta C_{l}(\varepsilon)<\frac{\varepsilon^{\prime}}{4}$. We thus have

$$
\left|\int_{0}^{\delta} \int_{\Omega} e_{m_{k}}\left(u_{m_{k}}-u^{*}\right) \Phi^{r} d x d t\right| \leq C_{s}(\varepsilon)+\delta C_{l}(\varepsilon) \leq \frac{\varepsilon^{\prime}}{2}
$$

Notice that

$$
\begin{aligned}
\left|\int_{\delta}^{T} \int_{\Omega} e_{m_{k}}\left(u_{m_{k}}-u^{*}\right) \Phi^{r} d x d t\right| & \leq C_{l}\left(\varepsilon^{\prime \prime}\right) \int_{\delta}^{T} \int_{\Omega}\left|e_{m_{k}}\left(u_{m_{k}}-u^{*}\right)^{2}\right| d x d t \\
& +C_{s}\left(\varepsilon^{\prime \prime}\right) \int_{\delta}^{T} \int_{\Omega}\left|e_{m_{k}}\left(\Phi^{r}\right)^{2}\right| d x d t \\
& \leq C_{l}\left(\varepsilon^{\prime \prime}\right)\left\{C_{l}\left(\varepsilon^{\prime \prime \prime}\right) \int_{\delta}^{T} \int_{\Omega}\left|\left(u_{m_{k}}-u^{*}\right)^{2}\right| d x d t\right. \\
& \left.+C_{s}\left(\varepsilon^{\prime \prime \prime}\right) \int_{\delta}^{T} \int_{\Omega}\left|\nabla\left(u_{m_{k}}-u^{*}\right)^{2}\right| d x d t\right\}+C_{s}\left(\varepsilon^{\prime \prime}\right) .
\end{aligned}
$$

We have

$$
\varlimsup_{k \rightarrow \infty}\left|\int_{\delta}^{T} \int_{\Omega} e_{m_{k}}\left(u_{m_{k}}-u^{*}\right) \Phi^{r} d x d t\right| \leq C_{l}\left(\varepsilon^{\prime \prime}\right) C_{s}\left(\varepsilon^{\prime \prime \prime}\right)+C_{s}\left(\varepsilon^{\prime \prime}\right) .
$$


For the $\varepsilon^{\prime}$ as before, we can choose $\varepsilon^{\prime \prime}$ such that $C_{s}\left(\varepsilon^{\prime \prime}\right)<\frac{\varepsilon^{\prime}}{4}$. Then for this fixed $\varepsilon^{\prime \prime}$, there exists an $\varepsilon^{\prime \prime \prime}$ such that $C_{l}\left(\varepsilon^{\prime \prime}\right) C_{s}\left(\varepsilon^{\prime \prime \prime}\right)<\frac{\varepsilon^{\prime}}{4}$. Hence

$$
\varlimsup_{k \rightarrow \infty}\left|\int_{\delta}^{T} \int_{\Omega} e_{m_{k}}\left(u_{m_{k}}-u^{*}\right) \Phi^{r} d x d t\right| \leq \frac{\varepsilon^{\prime}}{2} .
$$

Since $\varepsilon^{\prime}$ is arbitrary, by (3.5) and (3.6), we get $\left|\int_{Q} e_{m_{k}}\left(u_{m_{k}}-u^{*}\right) \Phi^{r} d x d t\right| \rightarrow 0$, as $k \rightarrow \infty$. The proof of Lemma 3.1 is complete.

Proof of Theorem 1.3. Let $d=\inf _{\left(e, a_{I}\right) \in \mathcal{M}_{q} \times \mathbf{R}^{\mathcal{K}}} \int_{Q^{\omega}}\left|u\left(e, a_{I} ; x, t\right)-\widetilde{u}\right|^{2} d x d t$. It is obvious that $d<\infty$. Thus there exists a sequence $\left\{\left(e_{m}, a_{I}^{m}\right)\right\}_{m=1}^{\infty}$ such that

$$
d \leq \int_{Q^{\omega}}\left|u_{m}\left(e_{m}, a_{I}^{m} ; x, t\right)-\widetilde{u}\right|^{2} d x d t \leq d+\frac{1}{m},
$$

and

$$
\begin{cases}\frac{\partial u_{m}\left(e_{m}, a_{I}^{m} ; x, t\right)}{\partial t}+L_{0} u_{m}\left(e_{m}, a_{I}^{m} ; x, t\right)+e_{m}(x, t) u_{m}\left(e_{m}, a_{I}^{m} ; x, t\right)=f(x, t), & \text { in } Q, \\ u_{m}\left(e_{m}, a_{I}^{m} ; x, t\right)=0, & \text { on } \Sigma, \\ \left(u_{m}\left(e_{m}, a_{I}^{m} ; \cdot, 0\right), X_{j}\right)=a_{j}^{m}, & j \leq \mathcal{K}, \\ \left(u_{m}\left(e_{m}, a_{I}^{m} ; \cdot, 0\right), X_{j}\right)=\left(u_{m}\left(e_{m}, a_{I}^{m} ; \cdot, T\right), X_{j}\right), & j>\mathcal{K} .\end{cases}
$$

In what follows, when there is no confusion of notation, we simply write $u_{m}(x, t)$ for $u_{m}\left(e_{m}, a_{I}^{m} ; x, t\right)$. By the definition of $\mathcal{M}_{q}$, there exists a subsequence $\left\{m_{k}\right\}$ and $e^{*} \in \mathcal{M}_{q}$ such that

$$
e_{m_{k}} \rightarrow e^{*} \text { in the weak star topology as } k \rightarrow \infty .
$$

By (3.7),

$$
\int_{Q^{\omega}}\left|u_{m}\right|^{2} d x d t \leq \int_{Q^{\omega}}|\widetilde{u}|^{2} d x d t+C \leq C
$$

with $C$ independent of $m$.

Next, we prove the following claim:

Claim 3.3. There is a constant $M_{0}$ such that $\left|a_{I}^{m}\right|^{2} \leq M_{0}$ for all $m$.

Proof of Claim 3.3. Suppose not . There is a subsequence $\left\{m_{k}\right\}$ such that $\mu_{k}=$ $\left|a_{I}^{m_{k}}\right| \rightarrow \infty$ as $k \rightarrow \infty$. We write $\hat{u}_{m_{k}}(x, t)=\frac{u_{m_{k}}(x, t)}{\mu_{k}}$. Then

$$
\begin{cases}\frac{\partial \hat{u}_{m_{k}}(x, t)}{\partial t}+L_{0} \hat{u}_{m_{k}}(x, t)+e_{m_{k}}(x, t) \hat{u}_{m_{k}}(x, t)=\frac{f(x, t)}{\mu_{k}}, & \text { in } Q, \\ \hat{u}_{m_{k}}(x, t)=0, & \text { on } \Sigma, \\ \left(\hat{u}_{m_{k}}(\cdot, 0), X_{j}\right)=\frac{a_{j}^{m_{k}}}{\mu_{k}}, & j \leq \mathcal{K}, \\ \left(\hat{u}_{m_{k}}(\cdot, 0), X_{j}\right)=\left(\hat{u}_{m_{k}}(\cdot, T), X_{j}\right), & j>\mathcal{K} .\end{cases}
$$


Apparently, $\left|\frac{a_{I}^{m_{k}}}{\mu_{k}}\right|^{2}=1$ for each $m_{k}$. After passing to a subsequence, if necessary, we can assume that

$$
\frac{a_{j}^{m_{k}}}{\mu_{k}} \rightarrow \hat{a}_{j} \text { with }\left|\hat{a}_{I}\right|=1, \hat{a}_{I}=\left(\hat{a_{1}}, \hat{a_{2}}, \cdots, \hat{a}_{\mathcal{K}}\right), j=1,2, \cdots, \mathcal{K} .
$$

Then by Lemma 3.1, there is a $\hat{u} \in C\left([0, T] ; L^{2}(\Omega)\right) \cap L^{2}\left(0, T ; H_{0}^{1}(\Omega)\right)$ such that $\hat{u}_{m_{k}} \rightarrow \hat{u}$ in the weak $L^{2}$-topology, and for any $\delta>0, \hat{u}_{m_{k}}(x, t) \rightarrow \hat{u}(x, t)$ strongly in the $L^{2}([\delta, T] \times \Omega)$ topology. Also, all the other statements in Lemma 3.1 hold.

By (3.7), we have

$$
\begin{aligned}
C & \geq \int_{Q^{\omega}}\left|u_{m_{k}}(x, t)-\widetilde{u}\right|^{2} d x d t \\
& =\int_{Q^{\omega}}\left|\mu_{k} \hat{u}_{m_{k}}(x, t)-\widetilde{u}\right|^{2} d x d t \\
& =\left(\mu_{k}\right)^{2} \int_{Q^{\omega}}\left|\hat{u}_{m_{k}}(x, t)-\frac{\widetilde{u}}{\mu_{k}}\right|^{2} d x d t \\
& \geq\left(\mu_{k}\right)^{2} \int_{[\delta, T] \times \omega}\left|\hat{u}_{m_{k}}(x, t)-\frac{\widetilde{u}}{\mu_{k}}\right|^{2} d x d t, \forall \delta>0 .
\end{aligned}
$$

Since $\mu_{k} \rightarrow \infty$, we see that

$$
\lim _{k \rightarrow \infty} \int_{[\delta, T] \times \omega}\left|\hat{u}_{m k}(x, t)-\frac{\widetilde{u}}{\mu_{k}}\right|^{2} d x d t=0, \forall \delta>0 .
$$

From the property that $\hat{u}_{m_{k}}(x, t) \rightarrow \hat{u}(x, t)$ strongly in the $L^{2}([\delta, T] \times \Omega)$, it follows that

$$
\int_{[\delta, T] \times \omega}|\hat{u}(x, t)|^{2} d x d t=0, \forall \delta>0 .
$$

By the unique continuation property for solutions of the parabolic equations, which is a consequence the Carleman inequality (see Page 430, Inequality (2) of [16]), we get $\hat{u}(x, t) \equiv 0$ in $(0, T] \times \Omega$. Since $\hat{u} \in C\left([0, T], L^{2}(\Omega)\right)$, we get $\hat{u}(x, 0) \equiv 0$. On the other hand, by $(3.12),\left(\hat{u}(\cdot, 0), X_{j}\right)=\hat{a}_{I}$ with $\left|\hat{a}_{I}\right|=1$. We see a contraction. The proof of Claim 3.3 is complete.

Now, making use of Claim 3.3, Lemma 3.1 and (3.9), we can assume that there is a subsequence $\left\{m_{k}\right\}$ such that $a_{I}^{m_{k}} \rightarrow a_{I}^{*},\left\{u_{m_{k}}\right\}$ converges in the weak $L^{2}$-topology to $u^{*} \in C\left([0, T] ; L^{2}(\Omega)\right) \cap L^{2}\left(0, T ; H_{0}^{1}(\Omega)\right)$ with

$$
\begin{cases}\frac{\partial u^{*}(x, t)}{\partial t}+L_{0} u^{*}(x, t)+e^{*}(x, t) u^{*}(x, t)=f^{*}(x, t), & \text { in } Q, \\ u^{*}(x, t)=0, & \text { on } \Sigma, \\ \left(u^{*}(\cdot, 0), X_{j}\right)=a_{j}^{*}, & j \leq \mathcal{K}, \\ \left(u^{*}(\cdot, 0), X_{j}\right)=\left(u^{*}(\cdot, T), X_{j}\right), & j>\mathcal{K} .\end{cases}
$$


By (3.7), we obtain

$$
d \leq \int_{Q^{\omega}}\left|u^{*}(x, t)-\widetilde{u}\right|^{2} d x d t \leq \lim _{k \rightarrow \infty} \int_{Q^{\omega}}\left|u_{m_{k}}(x, t)-\widetilde{u}\right|^{2} d x d t \leq d .
$$

So

$$
\int_{Q^{\omega}}\left|u\left(e^{*}, a_{I}^{*} ; x, t\right)-\widetilde{u}\right|^{2} d x d t=\inf _{\left(e, a_{I}\right) \in \mathcal{M}_{q} \times \mathbf{R}^{\mathcal{K}}} \int_{Q^{\omega}}\left|u\left(e, a_{I} ; x, t\right)-\widetilde{u}\right|^{2} d x d t .
$$

The proof of Theorem 1.3 is complete.

Proof of Corollary 1.4: By Theorem 1.3, we can assume that $k<\mathcal{K}_{0}$, where $\mathcal{K}_{0}$ is the same integer as in Theorem 1.2. Notice that $\mathcal{S}_{k} \subset \mathcal{S}_{\mathcal{K}_{0}}$. We keep the notation set up before.

Still let $d=\inf _{\left(e, a_{I}\right) \in \mathcal{M}_{q} \times \mathbf{R}^{k}, u \in U\left(e, a_{I} ; x, t\right)} \int_{Q^{\omega}}|u(x, t)-\widetilde{u}|^{2} d x d t$ with $d<\infty$. We then also have a sequence of the pairs $\left\{\left(e_{m}, a_{I}^{m}\right)\right\}_{m=1}^{\infty}$ and a sequence of functions $u_{m}\left(e_{m}, a_{I}^{m} ; x, t\right)$ such that

$$
d \leq \int_{Q^{\omega}}\left|u_{m}\left(e_{m}, a_{I}^{m} ; x, t\right)-\widetilde{u}\right|^{2} d x d t \leq d+\frac{1}{m}
$$

and

$$
\begin{cases}\frac{\partial u_{m}\left(e_{m}, a_{I}^{m} ; x, t\right)}{\partial t}+L_{0} u_{m}\left(e_{m}, a_{I}^{m} ; x, t\right)+e_{m}(x, t) u_{m}\left(e_{m}, a_{I}^{m} ; x, t\right)=f(x, t), & \text { in } Q, \\ u_{m}\left(e_{m}, a_{I}^{m} ; x, t\right)=0, & \text { on } \Sigma, \\ \left(u_{m}\left(e_{m}, a_{I}^{m} ; \cdot, 0\right), X_{j}\right)=a_{j}^{m} & j \leq k, \\ \left(u_{m}\left(e_{m}, a_{I}^{m} ; \cdot, 0\right), X_{j}\right)=\left(u_{m}\left(e_{m}, a_{I}^{m} ; \cdot, T\right), X_{j}\right), & j>k .\end{cases}
$$

Now, write $\widetilde{a}_{I}^{m}=\left(a_{1}^{m}, \cdots, a_{k}^{m}, \widetilde{a}_{k+1}^{m}, \widetilde{a}_{k+2}^{m}, \cdots, \widetilde{a}_{\mathcal{K}_{0}}^{m}\right)$ with $\widetilde{a}_{j}^{m}=\left(u_{m}\left(e_{m}, a_{I}^{m} ; x, 0\right), X_{j}\right)=$ $\left(u_{m}\left(e_{m}, a_{I}^{m} ; x, T\right), X_{j}\right)$ for $j=k+1, k+2, \cdots, \mathcal{K}_{0}$. Notice that

$$
\int_{Q^{\omega}}\left|u_{m}\left(e_{m}, a_{I}^{m} ; x, t\right)\right|^{2} d x d t \leq \int_{Q^{\omega}}|\widetilde{u}|^{2} d x d t+C \leq C
$$

Since $u_{m}\left(e_{m}, a_{I}^{m} ; x, t\right) \in \mathcal{S}_{k} \subset \mathcal{S}_{\mathcal{K}_{0}}$ for each $m$, making use of Lemma 3.1 , we can repeat the same argument as in the proof of Theorem 1.3 to show that there is a subsequence $\left\{u_{m_{l}}\left(e_{m_{l}}, a_{I}^{m_{l}} ; x, t\right)\right\}$ of $\left\{u_{m}\left(e_{m}, a_{I}^{m} ; x, t\right)\right\}$ such that $\widetilde{a}_{I}^{m_{l}} \rightarrow a_{I}^{*}$ and $\left\{u_{m_{l}}\right\}$ converges in the weak $L^{2}$-topology to $u^{*} \in C\left([0, T] ; L^{2}(\Omega)\right) \cap L^{2}\left(0, T ; H_{0}^{1}(\Omega)\right)$ with

$$
\begin{cases}\frac{\partial u^{*}(x, t)}{\partial t}+L_{0} u^{*}(x, t)+e^{*}(x, t) u^{*}(x, t)=f(x, t), & \text { in } Q \\ u^{*}(x, t)=0, & \text { on } \Sigma \\ \left(u^{*}(\cdot, 0), X_{j}\right)=a_{j}^{*}, & j \leq \mathcal{K}_{0} \\ \left(u^{*}(\cdot, 0), X_{j}\right)=\left(u^{*}(\cdot, T), X_{j}\right), & j>\mathcal{K}_{0} .\end{cases}
$$

Moreover, as in Lemma 3.1, we also have 


$$
u_{j}^{*}(0)=\left(u^{*}(\cdot, 0), X_{j}\right)=\lim _{m_{l} \rightarrow \infty} u_{j}^{m_{l}}(0)=\lim _{m_{l} \rightarrow \infty} u_{j}^{m_{l}}(T)=u_{j}^{*}(T) \text { for } j>k .
$$

Hence, $u^{*} \in \mathcal{S}_{k}$. Now, by the same argument as in the proof of Theorem 1.3, we conclude the proof of Corollary 1.4 .

Proof of Theorem 1.5: Keep the notation as in Theorem 1.5. Let $a_{I}^{1}, a_{I}^{2} \in \mathbf{R}^{\mathcal{K}}$ be such that

$$
d=\int_{Q^{\omega}}\left|u\left(e, a_{I}^{j} ; x, t\right)-\widetilde{u}\right|^{2} d x d t=\inf _{a_{I} \in \mathbf{R}^{\mathcal{K}}} \int_{Q^{\omega}}\left|u\left(e, a_{I} ; x, t\right)-\widetilde{u}\right|^{2} d x d t, \quad j=1,2 .
$$

For $\tau \in \mathbf{R}$, define

$$
\begin{gathered}
I(\tau)=\int_{Q^{\omega}}\left|u\left(e, \tau a_{I}^{1}+(1-\tau) a_{I}^{2} ; x, t\right)-\widetilde{u}\right|^{2} d x d t \text {. Then } \\
I(\tau)=\int_{Q^{\omega}}\left|\tau\left(u\left(e, a_{I}^{1} ; x, t\right)-\widetilde{u}\right)+(1-\tau)\left(u\left(e, a_{I}^{2} ; x, t\right)-\widetilde{u}\right)\right|^{2} d x d t . \\
=d\left(\tau^{2}+(1-\tau)^{2}\right)+2 \tau(1-\tau) \int_{Q^{\omega}}\left(u\left(e, a_{I}^{1} ; x, t\right)-\widetilde{u}\right)\left(u\left(e, a_{I}^{2} ; x, t\right)-\widetilde{u}\right) d x d t .
\end{gathered}
$$

Since $I(\tau)$ achieves its minimum value at $\tau=0$, we have $I^{\prime}(0)=0$, from which the following follows:

$$
d=\int_{Q^{\omega}}\left(u\left(e, a_{I}^{1} ; x, t\right)-\widetilde{u}\right)\left(u\left(e, a_{I}^{2} ; x, t\right)-\widetilde{u}\right) d x d t .
$$

On the other hand, by the Hölder inequality, we have

$$
\begin{gathered}
\left(\int_{Q^{\omega}}\left(u\left(e, a_{I}^{1} ; x, t\right)-\widetilde{u}\right)\left(u\left(e, a_{I}^{2} ; x, t\right)-\widetilde{u}\right) d x d t\right)^{2} \\
\leq \int_{Q^{\omega}}\left|u\left(e, a_{I}^{1} ; x, t\right)-\widetilde{u}\right|^{2} d x d t \cdot \int_{Q^{\omega}}\left|u\left(e, a_{I}^{2} ; x, t\right)-\widetilde{u}\right|^{2} d x d t=d^{2},
\end{gathered}
$$

with equality being held if and only if $\left(u\left(e, a_{I}^{1} ; x, t\right)-\widetilde{u}\right)=C\left(u\left(e, a_{I}^{2} ; x, t\right)-\widetilde{u}\right)$ over $Q^{\omega}$ for a certain constant $C$. Apparently, this implies that

$$
u\left(e, a_{I}^{1} ; x, t\right)-\widetilde{u}=u\left(e, a_{I}^{2} ; x, t\right)-\widetilde{u} \text { over } Q^{\omega} .
$$

By the Carleman inequality as mentioned in the proof of Theorem 1.3, we conclude that $u\left(e, a_{I}^{1} ; x, t\right)=u\left(e, a_{I}^{2} ; x, t\right)$ over $Q$. This then forces that they have the same initial value. In particular we conclude that $a_{I}^{1}=a_{I}^{2}$. The proof of Theorem 1.5 is complete.

Example 3.4: Consider the following heat equation:

$$
y_{t}=\Delta y+c y+f(x, t), \quad 0 \leq x \leq 1, \quad 0 \leq t \leq 1
$$


Here $c \in \mathbf{R}$ and $f \in L^{2}((0,1) \times(0,1))$. Notice that $\left\{\frac{1}{\sqrt{2}} \sin (k \pi x)\right\}_{k=1}^{\infty}$ forms an orthonormal basis of $L^{2}(0,1)$. Write $f(x, t)=\sum_{k=1}^{\infty} f_{k}(t) \sin (k \pi x)$ and $y=\sum_{k=1}^{\infty} a_{k}(t) \sin (k \pi x)$. Then we have $a_{k}^{\prime}(t)=-(k \pi)^{2} a_{k}(t)+c a_{k}(t)+f_{k}(t)$. Thus, we get

$$
a_{k}(1) \mathrm{e}^{-c+(k \pi)^{2}}-a_{k}(0)=\int_{0}^{1} f_{k}(t) \mathrm{e}^{\left(-c+(k \pi)^{2}\right) t} d t .
$$

Now, choose $c=(K \pi)^{2}$ and choose $f$ such that $\int_{0}^{1} f_{K}(t) d t \neq 0$. Then (3.16) can never have a solution $y$, which is in the space $\mathcal{S}_{K-1}$. However, in this case, for any $b_{I}^{K}=\left(b_{1}, \cdots, b_{K}\right)$, (3.16) does have a unique solution $y(x, t) \in \mathcal{S}_{K}$ with $\left(y(\cdot, 0), \frac{1}{\sqrt{2}} \sin (j \pi x)\right)=b_{j}$ for $j=$ $1,2, \cdots, K$.

\section{References}

[1] H.T. Bank and K. Kunisch, Estimation Techniques for Distributed Parameter Systems, Boston, MA: Birkhäuser, 1989.

[2] V. Barbu, Controllability of parabolic and Navier-Stokes equations, Scientia Mathematica Japonica, 6(2002), pp: 143-211.

[3] V. Barbu, Analysis and Control of Nonlinear Infinite Dimensional Systems, Academic Press, New York, NY, 1993.

[4] J. Cannon, The One-dimensional Heat Equation (Encyclopedia of Mathematics 23), London: Addison-Wesley, 1984.

[5] Ya-Zhe Chen, Parabolic Partial Differential Equations of Second Order, Series in Graduate Textbooks in Mathematics, Beijing University Press, Beijing, January, 2003.

[6] H.W. Engl, M. Hanke and A. Neubauer, Regularization of Inverse Problems, Dordrecht: Kluwer, 1996.

[7] H.W. Engl and J. Zou, A new approach to convergence rate analysis of Tikhonov regularization for parameter identification in heat conduction, Inverse Problems, 16, pp: 1907-1923, 2000.

[8] D. Gilbarg and N. S.Trudinger, Elliptic Partial Differential Equations of Second Order. Grundlehren der mathematischen Wissenschaften, a Series of Comprehensive Studies in Mathematics, Vol. 224, 2. Edition, Springer 1977, 1983

[9] S. Gutman, Identification of discontinuous parameters in flow equations, SIAM J. Control Optim., 28, pp: 1049-1060, 1990. 
[10] O.Yu. Imanuvilov, Controllability of parabolic equations, Sb. Math., 186(6), pp: 879900, 1995.

[11] C. Jia and G. Wang, Identifications of parameters in ill-posed linear parabolic equations, Nonlinear Analysis, Vol57/5-6, p:677-686, 2004.

[12] Y.L. Keung and J. Zou, Numerical identifications of parameters in parabolic systems, Inverse Problems, 14, pp: 83-100, 1998.

[13] K. Kunisch and L. White, The parameter estimation problem for parabolic equations and discontinuous observation operators, SIAM J. Control Optim., 23, pp: 900-927, 1985.

[14] L. Lei, Identification of Parameters in Periodic Parabolic Equations, Journal of Mathematics (Wuhan), 2005. (to appear)

[15] G. Wang, Optimal control of parabolic partial differential equations with two pointpoint boundary state constraint, SIAM Journ on Control and Optimazation, Vol. 38, 2000 .

[16] G. Wang and L. Wang, The Carleman inequality and its application to periodic optimal control governed by parabolic system, Journal of Optimization Theory and Application, Vol. 118. No.2, pp: 429-461, 2003.

[17] G. Wang and L. Wang, Identification of Nonlinearity in Periodic Parabolic Equations, Numerical Functional Analysis and Optimization, Vol.25(1-2), pp: 183-197, 2004.

Regular Mailing Address: Room 5032, Dormitory 29, Yuquan Campus, Zhejiang University, Hangzhou 310027, P. R. China

E-mail Address: leiling0810@yahoo.com.cn 